\title{
A Critical Review on Traditional Herbal Drugs: An Emerging Alternative Drug for Diabetes
}

\author{
Krishna Bihari Pandeya ${ }^{1}$, Indra Prasad Tripathi ${ }^{2 *}$, Mahendra Kumar Mishra ${ }^{3 *}$, Neelesh Dwivedi ${ }^{3}$, \\ Yogesh Pardhi ${ }^{4}$, Arti Kamal ${ }^{3}$, Priyanka Gupta ${ }^{3}$, Nupa Dwivedi ${ }^{3}$, Chinmayi Mishra ${ }^{3}$ \\ ${ }^{1}$ Vice-Chancellor, Mahatma Gandhi Chitrakoot Vishwavidyalaya, Chitrakoot, India \\ ${ }^{2}$ Faculty of Science \& Environment, Mahatma Gandhi Chitrakoot Gramoday Vishwavidyalaya, Chitrakoot, India \\ ${ }^{3}$ Research Scholar, Mahatma Gandhi Chitrakoot Vishwavidyalaya, Chitrakoot, India \\ ${ }^{4}$ Reseach Assistant, Tropical Forest Research Institute (TFRI), Jabalpur, India \\ Email: "tripathi.ip@gmail.com
}

Received September 7, 2012; revised November 2, 2012; accepted January 17, 2013

\begin{abstract}
Diabetes is a chronic metabolic disease reaching an epidemic proportion in many parts of the world. By the year 2025 it is expected that 333 million people of the world will have diabetes as their main ailment. As today, India assumes the position of the diabetic capital of the world with the highest percentage of its population suffering from diabetes. It is pathetic to mention that in proportion to its people suffering from diabetes, this country has very weak spending power for treatment because of wide spread poverty. Therefore, this review is aimed at opening up new vistas in realizing the therapeutic potential of Ayurveda in treatment of diabetes and other chronic diseases. All drugs which we have discussed in this review have a significant role in therapy of diabetes mellitus.
\end{abstract}

Keywords: Diabetes Mellitus; Metabolic Disease; Ayurveda; Hyperglycemia; Synthetic Drugs; Herbal Drugs; Metallo Therapy; Oral Hypoglycemic Drugs

\section{Introduction}

The word diabetes was coined by the Greek physician Aretaeus in the first century AD Diabetes mellitus has been known since ages and sweetness of urine has been mentioned in Ayurveda by Sushruta. Its pharmacotherapy is 80 year old. The presence of sugar in the urine of diabetics was demonstrated by Dobson in 1755 [1]. Yet, as we have spent one decade of new millennium, our knowledge of the nature and treatment of diabetes is still incomplete. Diabetes mellitus is most serious; chronic metabolic and characterized by high blood glucose level [2]. Hyperglycemia is caused by relative or absolute deficiency of insulin or by a resistance to the action of insulin at the cellular level. It is most common endocrine disorder, affecting 41 million individuals in India and as many as $\mathbf{2 0 0}$ million world-wide. The worldwide prevalence of diabetes for all age groups was estimated to be $2.8 \%$ in 2000 and it is projected to be $5.4 \%$ in 2025 . Currently available therapies for diabetes include insulin and oral antidiabetic agents such as sulfonylurea, biguanides, $\alpha$-glucosidase inhibitors and glinides, in develpoping countries as products are expensive and not easily accessible [3]. DM (diabetes mellitus) has been become a

\footnotetext{
"Corresponding author.
}

clinical model for general model for general medicine. The primary defect in fuel metabolism results in widespread, multi-organ complications that ultimately encompass virtually every system of the body. Although from a clinical standpoint, this may be true, our increasing knowledge of patho-physiology of the syndrome, together with the mechanisms of long term complication, has placed diabetes researched at the frontier of immunology and molecular biology [1].

\section{Etiology of Diabetes, Cure and Strategy}

Diabetic patients are diagnosed by blood or urinary glucose measurement through different techniques. On the basis of etiology DM (Diabetes mellitus) are categories mainly two types viz:

1) Primary Diabetes (Type I or Insulin Dependent Diabetes Mellitus).

2) Secondary Diabetes (Type II or Non Iinsulin Dependent Diabetes Mellitus).

Primary DM (Diabetes mellitus) clinically dependent on insulin due to there is decrease in the number of $\beta$-cells in the islets of langerhans and thus there is absolute deficiency of insulin hence this is known as Insulin Dependent Diabetes Mellitus (IDDM) or Type I. The main treatment for this Type I of DM (Diabetes mellitus) 
is insulin.

Secondary DM (Diabetes mellitus) is referred as Type II or Non Insulin Dependent Diabetes Mellitus (NIDDM) because these types of patients are insulin resistances as well as loss of insulin secretion contributes to the onset of disease. The patients are usually obese and the treatment is usually dietary, through supplementary oral Hypoglycemic drugs.

As the complications induced by diabetes mellitus are very serious and goes to worst day by day hence, there is potent need of medicine to cure the diabetes mellitus. In this review we will go through the all the possible cure available to preventing the complications and its limitation also, to finding out the alternatives of these synthetic drugs, having no or few side effects with low price and ease to reach the common people.

\section{Strategy for Treatment of Diabetes}

Basic therapeutic approach to treat diabetes may be to inhibit the absorption of glucose by retarding the action of gastro-intestinal enzymes such as $\alpha$-glucosidase and $\alpha$-amylase. Because the complication of disease is mainly due to the higher glucose level in blood which dysfunction the other organs of body. Thus we can say that the effective $\alpha$-glucosidase inhibitors may serves as chemotherapeutic agents for clinic use in the treatment of diabetes and obesity $[4,5]$.

\subsection{Medicine for Treatment of Diabetes Mellitus}

\subsubsection{Insulin}

Insulin increases glucose uptake in cells by stimulating the translocation of the glucose transporter GLUT4 from intracellular sites to the cell surface [6,7]. Insulin circulates in blood as the free monomer and its half life in plasma is about $5-6 \mathrm{~min}$ in normal subjects. Although glucose is the principal stimulus to insulin secretion in human beings, this process is tightly regulated by the coordinated of nutrients, gastrointestinal and pancreatic hormones and autonomic neurotransmitters [8]. The main draw back of insulin is taken through injection.

\subsubsection{Oral Hypoglycemic Drugs}

Oral Hypoglycemic drugs are those drugs that lower blood glucose level and taken orally. These drugs are synthetic and complex organic substances. Hence the search for oral active drugs is in demand.

\section{1) Sulfonylureas Drugs}

First Generation Drugs

a) Tolbutamine;

b) Chloropropamide;

c) Acetohexamide;

d) Tolazmde.

Second Generation Drugs a) Gilbenclamide;

b) Glipizide;

c) Gliclazide.

2) Biguanides

a) Phenformin;

b) Metformin.

3) Others

a) Acarbose;

b) Guar Gum.

These drugs are effective in diabetes but having some limitations such as hypoglycemia occurs with regular use of sulfonylurea compounds but occurrences are much fewer than with insulin therapy. It is prescribed by doctors that biguanids should not use in patients with renal diseases. On the other hand the main side effect of Acarbose is flatulence [1].

\subsubsection{Metallotherapy}

The current literature also shows that metallopharmaceuticals is an area of growing interest as is evident through the clinical trials that are being conducted worldwide for the usages of metals in therapeutics. Metallotherapy is a new therapeutic strategy to treat diabetes with metal complexes. It is first studied by Coulson and Dandona in 1980 that $\mathrm{ZnCl}_{2}$ stimulate lipogenesis in rat adipocytes similarly to the action of Insulin. In three decades there are many researchers reported insulin-mimetic activity, $\alpha$-glucosidase and $\alpha$-amylase inhibition with different coordination of different ligand with transition metals. However the strategies to treat the diabetes mellitus through metal complexes are in early stage hence there are no side effects reported at all. Some metal complexes are in trial stage for treating diabetes.

\subsubsection{Herbal Drugs}

There are many herbal products/herbal extracts are reported to treat the diabetes mellitus, we can classify these drugs according to their mode of action as:

\subsubsection{Extracts/Drugs Act as $\alpha$-Glucisidase or $\alpha$-Amylase Inhibitor}

These types of drugs/extracts are able to reduce the blood glucose level by inhibiting the gastric enzymes which is obligatory for the break the polysaccharides in to the simple sugar.

The aqueous and methanolic extract of Syzgium cumini (seed) and Pisidium guajava (leaves) shows $\alpha$-amylase inhibition [9]; while Rhus verniciflua stem screened for $\alpha$-glucosidase inhibition effect mixture of methanol and ethanol extract shows the potent inhibition of $\alpha$-glucosedase enzyme [10]. There are large number of plants which have the capability to inhibit the $\alpha$-glucosidase and $\alpha$-amylase activity and may be used as treatment of diabetes Type I and Type II. 


\subsubsection{Extracts/Drugs Increases Insulin Secretion or $\beta$-Cell Regeneration}

These types of drugs are directly concern with the Type I or IDDM diabetes which are disable to secreting the less or few amount of insulin.

Radix of Acorus calamus is used as in the therapy of diabetes in traditional folk medicine of America and Indonesia, this sensitize the insulin activity of its ethyl acetate extract [11]. On the other hand Ginsenoside $\mathrm{Rh} 2$ an active compound found in Panax ginseng root increased plasma insulin level parallel with lowering the plasma glucose level [12]. Moreover aqueous extract of Syzgium cumini bark stimulate $\beta$-cell regeneration by proliferation of its precursor or cells in the pancreatic duct [13].

\subsubsection{Extracts/Drugs Act as Hypoglycemic,}

Antihyperglycemic or Antidiabetic Effect

These classes of herbal drugs reduce the blood glucose level directly, this may be also used to treat the both type of diabetes mellitus (IDDM and NIDDM).

Mangifera indica Linn. (Locally known as mango tree) has antidiabetic property, ethanolic and water extract of leaves and stem bark of Mangifera indica shows significant antihyperglycemic effect [14]. Extract of Hedychium spicatum rhizomes also show the antihyperglycemic effect [15]. Ficus bengalensis bark extract show antidiabetic and ameliorative activity [16]. While alcoholic aqueous extract of Coccinia indica (C. cordifolia) aerial part exhibit the hypoglycemic effect and fruit of Ficus glomerata shows hypoglycemic activity $[17,18]$.

\subsubsection{Extracts/Drugs Dealing with the Complications of Diabetes Mellitus}

Diabetes mellitus is metabolic syndrome characterized by deregulation in carbohydrate metabolism associated with defect in insulin secretion or action by which glucose level of blood increases, the different type of complication occurred. To treat these type of problem many herbal drugs/extract may play a key role.

The aqueous extract of bark of Ficus religiosa Linn reduces oxidative stress in Type II diabetes mice model [19]. Extract of fruit of Benincasa gispida decrease gastric ulcer index in diabetic rat model [20]. Hexane extract of Derris scanders show potent $\alpha$-glucosidase inhibition effect and moderate free radical scavenging activity [21]. Pongamia pinnata flower shows antihyperglycemic and antilipid-peroxidative effect with reference drugs glibenclamide [22]. Oral administrations of Coccinia indica leaf extract ( $200 \mathrm{mg} / \mathrm{kg}$ body weight) for 45 days significantly reduce the thiobarbituric acid reactive substances and hydroperoxides. The extract also causes a significant increase in reduced glutathione, superoxide dismutase, catalase, glutathione peroxidase and glutathione-S-transferase in liver and kidney of streptozoto- cin diabetic rats, which clearly shows the antioxidant property [23]. Some plants are listed in Table 1 which has the antidiabetic properties.

\section{Plants Used for Curing Diabetes Mellitus in Ayurveda}

India has a great ancient heritage of traditional medicine. The material medica of Indian provides much information on ethnic folklore practices and traditional aspects of therapeutically important natural products $[26,27]$. Indian traditional medicine is based on various systems, including Ayurveda, Siddha and Unani (ASU). With the emerging interest of the world in adopting and studied traditional systems and in exploiting their potential from different healthcare perspectives, it is necessary to listing some of plants which are used to curing the diabetes from ancient time are listed in [28-31] Table 2.

\section{Trials of Traditional Medicines}

There are several herbal drugs are formulated according to traditional and modern knowledge of ethanobotany. Bio-active molecules have been cross checked on diabetic models and several are in trail courses. Some of trails are as:

Wendell D. Winters (2003) et al. [32] studied inhibittion of the progression of Type II diabetes in the C57BL/ $6 \mathrm{~J}$ mouse model by an anti-diabetes herbal formula prepare from Chinese herbs which was activated and mixed according to proprietary formula ingredients through standard method. The herbal formula had eight major herbals, Ginseng Radix (17\%), Rehmannia Radix (17\%), Astragali Radix (10\%), Trichosanthis Radix (10\%), Ophiopogous Radix (10\%), Puerariae Radix (10\%), Lycii fructus $(10 \%)$, Discoreae Rizoma (10\%) and found that $4 \%-8 \%$ of regular feeding with ADHF shows significant reduction in blood glucose level and increase in insulin level further they suggested ADHF should be used as diet supplement.

Ikuko Kimura (1999) et al. [33] studied the antihyperglycemic blend effect of traditional Chinese medicine byakko-ka-ninjin-to on alloxan and diabetic $\mathrm{KK}^{-\mathrm{CA}^{\mathrm{y}}}$ Mice and concluded that the water extract of Byakko-Kaninjin-to (BN) which have Ginseng root, licorice root, Anemarrhena asphodeloides (rhizome), Fibrosum gypsum and rice with blend of $\mathrm{Ca}^{++}$shows the antihyperglycemic effect.

In the management of diabetes Type II a trail of Vijayasar (Pterocarpus marsupium) had done in different location in India by ICMR group to check the efficacy of Vijayasar in contrast of synthetic drug Tolbutamide. Study shows that Vijayasar is an effective blood glucose lowering traditional Indian plant agent, its glycemic effect being comparable to that of Tolbutamide in treatment 
Table 1. Representing list of antidiabetic plants.

\begin{tabular}{|c|c|c|c|c|c|}
\hline S. N. & Plant name (botanical) & Family & Part(s) used & Effects observed & Reference(s) \\
\hline 1 & Abroma augusta & Sterculiaceae & Root & Hypoglycemic and antilipidemic & [40] \\
\hline 2 & Acacia arabica & Fabeceae & Bark & $\begin{array}{l}\text { Ameliorate the dearrangmrnt in lipid } \\
\text { metabolism in diabetes }\end{array}$ & [41] \\
\hline 3 & Acalypha wilkesiana & Euphorpiaceae & Leaves & Hypoglycemic and antioxidant & [42] \\
\hline 4 & Acer saccharum (maple) & Sapindaceae & Juice & $\alpha$-Amylase and $\alpha$-glucosidase inhibition & [43] \\
\hline 5 & Acorus calamus & Acoraceae & Leaves/bark & $\alpha$-Glucosidase inhibition and hypolipidemic & [11] \\
\hline 6 & Aegle marmelos & Rutaceae & Leaves/bark/root & Antioxidant and anti cataract activity & [44] \\
\hline 7 & Afzelia africana & Fabaceae & Stem bark & Antidiabetic and hematological effect & [45] \\
\hline 8 & Alhagi camelorum & Fabaceae & Aerial part & $\alpha$-Glucosidase inhibition & [46] \\
\hline 9 & Allium sattivum & Alliaceae & Bulb & Antidiabetic & [3] \\
\hline 10 & Aloe vera & Xanthorrhocaceae & Leaves & Hypoglycemic & [47] \\
\hline 11 & Alpinia officinarum & Zingiberaceae & Rhizomes & $\alpha$-Glucosidase inhibition & [46] \\
\hline 12 & $\begin{array}{l}\text { Amaranthus spinosus, } \\
\text { Amaranthus caudatus, } \\
\text { Amaranthus viridis }\end{array}$ & Amaranthaceae & Leaves & $\begin{array}{l}\alpha \text {-Amylase inhibition, antioxidant and } \\
\text { anticholesrolemic }\end{array}$ & {$[48,49]$} \\
\hline 13 & Annona squamosa & Annonaceae & Fruits & Antidiabetic & [3] \\
\hline 14 & Anogeissus acuminate & Combretaceae & Whole plant & Antioxidant & [50] \\
\hline 15 & Areca catechu & Arecaceae & Seed & Antidiabetic & [3] \\
\hline 16 & $\begin{array}{l}\text { Artemis sphaerocephala } \\
\text { krasch }\end{array}$ & Compositae & Gum/seed powder & Antidiabetic & [51] \\
\hline 17 & Artemisia dracunculus (L.) & Asteraceae & Aerial part & Antidiabetic & [52] \\
\hline 18 & Artemisia herba-alba asso & Asteraceae & Whole plant & Antihyperglycemic and antihyperlipidemic & [53] \\
\hline 19 & Artemisia pallens & Asteaceae & Leaves and Flower & Antidiabetic & [3] \\
\hline 20 & Ascophyllum nodosum & Algae & - & $\alpha$-Glucosidase inhibition and $\alpha$-amylase & [54] \\
\hline 21 & Aspidosperma macrocarpon & Apocynaceae & Steam/Bark Root wood & $\alpha$-Amylase inhibition & [55] \\
\hline 22 & Atractylodes macrocephala & Asteraceae & Whole plant & $\alpha$-Glucosidase inhibition & {$[56]$} \\
\hline 23 & Azadirachta indica & Meliaceae & $\begin{array}{l}\text { Leaves, flower } \\
\text { and seed }\end{array}$ & Antidiabetic and antihypertension & {$[3,57,58]$} \\
\hline 24 & Barringtonia racemosa & Lecythidaceae & Seed & $\alpha$-Glucosidase and $\alpha$-amylase inhibition & {$[59,60]$} \\
\hline 25 & Bauhinia forficate & Leguminosae & Leaves & Antidiabetic & [3] \\
\hline 26 & Belamcanda chinensis & Irdaceae & Leaves & Hypoglycemic & {$[61,62]$} \\
\hline 27 & Benincasa hispida & Cucurbitaceae & Fruit & $\begin{array}{l}\text { Ameliorat the derangement in lipid } \\
\text { metabolism in diabetics. }\end{array}$ & [41] \\
\hline 28 & Benincasa hispida & Cucurbitaceae & Fruit & Antidiabetic & {$[20,63]$} \\
\hline 29 & Berberis vulgaris & Berberidaceae & Leaves & Hypoglycemic & [64] \\
\hline 30 & Beta vulgaris & Amaranthaceae & Root & Antidiabetic & [3] \\
\hline
\end{tabular}




\section{Continued}

\begin{tabular}{|c|c|c|c|c|c|}
\hline 31 & Borerhavia diffusa & Nyctaginaceae & Whole plant & Antidiabetic & {$[3]$} \\
\hline 32 & Brassica juncea & Brassicaceae & Seed & Hypoglycemic & {$[65]$} \\
\hline 33 & Buchholzia coriaceae & Capparaceae & Seed & Hypoglycemic & {$[66]$} \\
\hline 34 & Caesalpinia ferrea Martius & Leguminosae & Bark & Hypoglycemic activity & {$[67]$} \\
\hline 35 & Camellia sinensis & Theaceae & Fruit peel/leaves & $\begin{array}{l}\alpha \text {-glucosidase and } \alpha \text {-amylase inhibition, } \\
\text { antihyperglycemic }\end{array}$ & {$[3,68,69]$} \\
\hline 36 & Capparis deciduas & Capparaceae & Fruit & Hypoglycemic/antidiabetic & {$[3,70]$} \\
\hline 37 & Cassia auriculata & Caesalpiniaceae & Aerial part & Antioxidant & {$[71]$} \\
\hline 38 & Catharanthus roseus & Apocynaceae & Leaves/bark/root & Antioxidant and anticataract activity & {$[44,72]$} \\
\hline 39 & Catunaregam tormentosa & Rubiaceae & Whole plants & Antioxidant and antihyperglycemic & {$[50]$} \\
\hline 40 & Centauriun erythrea & Gentianaceae & Whole plant/leaves & $\begin{array}{l}\text { Antihyperglycemic, antihyperlipidemic, } \\
\text { antioxidant and prevent } \beta \text {-cell damage }\end{array}$ & {$[53,73]$} \\
\hline 41 & Chaenomeles sinensis & Rosaceae & Fruit & $\begin{array}{c}\alpha \text {-Glucosidase and } \beta \text {-glucosidase } \\
\text { inhibition }\end{array}$ & {$[74]$} \\
\hline 42 & Chiliadenus iphionoides & Asteraceae & Shrub & $\begin{array}{l}\text { Increased } \beta \text {-cells numbers and } \\
\text { Insulin secretion }\end{array}$ & {$[75]$} \\
\hline 43 & $\begin{array}{l}\text { Cinnamomum cassia, } \\
\text { Cinnamomum zeylanicum }\end{array}$ & Lauraceae & Leaves/bark & $\begin{array}{c}\alpha \text {-Glucosidase inhibition, antioxidant } \\
\text { and antidiabetic }\end{array}$ & {$[3,56,76]$} \\
\hline 44 & Coccinia cordifolia & Cucurbitaceae & Herb & Hypoglycemic & {$[17]$} \\
\hline 45 & Coccinia indica & Cucurbitaceae & Leaves & $\begin{array}{l}\text { Antioxidant, antihyperglycemic } \\
\text { and antilipidemic }\end{array}$ & {$[23,24,40]$} \\
\hline 46 & Cocos nucifera & Arecaceae & Coconut kernel protein & Antidiabetic & {$[77]$} \\
\hline 47 & Codonopsis pilosula & Campanulaceae & Whole plant & $\alpha$-Glucosidase inhibition & {$[56]$} \\
\hline 48 & $\begin{array}{l}\text { Coffea arabica, } \\
\text { Coffea canephora }\end{array}$ & Rubiaceae & Beans/seed & $\alpha$-Amylase isoenzyme inhibition & {$[78,79]$} \\
\hline 49 & Combretum micranthum & Combretaceae & Leaves & Antidiabetic & {$[3]$} \\
\hline 50 & Commbretum micranthum & Combretaceae & Leaves & Hypoglycemic and antidiabetic & {$[3,80]$} \\
\hline 51 & Commiphora mukul & Burseraceae & Gum resin & Antihyperglycemic and antioxidant & {$[81]$} \\
\hline 52 & Coriandrum sativum & Apiaceae & Seed & Hypolipidemic and hypoglycemic & {$[82]$} \\
\hline 53 & Corus kousa & Cornaceae & Leaves & Antihyperglycemic & {$[83]$} \\
\hline 54 & Costus pictus & Zingiberaceae & Leaves & $\alpha$-Glucosidase and $\alpha$-amylase inhibition & {$[84]$} \\
\hline 55 & $\begin{array}{l}\text { Cucumis melo var. } \\
\text { utlissimus Duthie }\end{array}$ & Cucurbitaceae & Fruit & Antioxidant and hyperglycemic & {$[63]$} \\
\hline 56 & Cucurbita maxima & Cucurbitaceae & Fruit & Antioxidant and hyperglycemic & {$[63]$} \\
\hline 57 & Cuminum cyminum & Apiaceae & Seed & $\begin{array}{l}\text { Aldose reductase and } \alpha \text {-glucosidase } \\
\text { inhibition }\end{array}$ & {$[85]$} \\
\hline 58 & Cynodon dactylon & Poaceae & Leaves & $\begin{array}{c}\text { Antidiabetic, antioxidant, hypolipidemic } \\
\text { and improve diabetes associated } \\
\text { neurological disorder }\end{array}$ & {$[86,87]$} \\
\hline 59 & Derris scandens & Fabaceae & Whole plant & $\alpha$-Glucosidase inhibition & [21] \\
\hline
\end{tabular}




\section{Continued}

\begin{tabular}{|c|c|c|c|c|c|}
\hline 60 & Dichrostachys glomerata & Fabaceae & Whole plant & Antioxidant and hypoglycemic & [88] \\
\hline 61 & Dillenia indica & Dilleniaceae & Leaves & Antidiabetic and hypolipidemic & [89] \\
\hline 62 & Dioecrescis erythroclada & Rubiaceae & Whole plant & Antioxidant and antihyperglycemic & [50] \\
\hline 63 & Dioscorea opposite & Dioscoreaceae & Leaves & $\alpha$-Glucosidase inhibition & [56] \\
\hline 64 & $\begin{array}{l}\text { Elephantopus scaber, } \\
\text { Elephantopus mollis }\end{array}$ & Asteraceae & Whole plant & $\begin{array}{l}\text { Antidiabetic, } \alpha \text {-glucosidase inhibition } \\
\text { and apoptotic }\end{array}$ & {$[3,90]$} \\
\hline 65 & Eleutherine americana & Iridaceae & Bud & $\alpha$-Glucosidase inhibition & [91] \\
\hline 66 & $\begin{array}{l}\text { Embelia ribes, } \\
\text { Embelia officinalis }\end{array}$ & Myrsinaceae & Leaves/aerial part & Antihyperglycemic & {$[92,93]$} \\
\hline 67 & Eriobotrya japonica & Rosaceae & Leaves & Antihyperglycemic & [94] \\
\hline 68 & Euclea undulata & Ebenaceae & Root/bark & $\alpha$-Glucosidase inhibition and hypoglycemic & [95] \\
\hline 69 & Eucommia ulmoides & Eucommiaceae & Whole plant & $\begin{array}{c}\alpha \text {-Glucosidase inhibition, antidiabetic } \\
\text { and antioxidant }\end{array}$ & [56] \\
\hline 70 & Ficus bengalensis Linn & Moraceae & Bark & $\begin{array}{l}\text { Antidiabetic, antihyperglycemic and } \\
\text { ameliorative }\end{array}$ & {$[3,16,96]$} \\
\hline 71 & Ficus golmerata & Moraceae & Bark/fruit & Antioxidant, anti cataract activity & {$[18,44]$} \\
\hline 72 & Ficus religiosa & Moraceae & Bark & $\begin{array}{l}\text { Antihyperglycemic, antioxidant and } \\
\text { antidiabetic }\end{array}$ & [19] \\
\hline 73 & Flos ionicerae & Caprifoliaceae & Whole plant & $\alpha$-Glucosidase inhibition & [56] \\
\hline 74 & Foenum graecum & Fabaceae & Whole plant & Antihyperglycemic & [97] \\
\hline 75 & Glycine $\max$ & Fabaceae & Seed & $\alpha$-Amylase inhibition & [98] \\
\hline 76 & Grateloupia elliptica & Algae & - & $\alpha$-Glucosidase inhibition & [99] \\
\hline 77 & Gyanandropsis gynandra & Capparidceae & Root & Antidiabetic & [3] \\
\hline 78 & $\begin{array}{l}\text { Gymnema sylvestre, } \\
\text { Gymnema montanum }\end{array}$ & Asclepiadaceae & Leaves/callus/stem & $\begin{array}{l}\text { Regeneration of } \beta \text { cell, reduce blood } \\
\text { glucose level, increase plasma insulin } \\
\text { level and hypolipidemic }\end{array}$ & {$[3,100-102]$} \\
\hline 79 & Gynura divaricata & Asteraceae & Aerial part & $\begin{array}{c}\text { Hypoglycemic, } \alpha \text {-glucosidase } \\
\alpha \text {-amylase inhibition }\end{array}$ & [103] \\
\hline 80 & Hedychium spicatum & Zingiberaceae & Rhizome & $\begin{array}{l}\alpha \text {-Glucosidase inhibition and } \\
\text { antihyperglycemic }\end{array}$ & {$[15]$} \\
\hline 81 & Helicteres igora & Sterculiaceae & Bark & Antihyperglycemiv and antiperoxidative & [104] \\
\hline 82 & Helleborus purpurascens & Ranunculaceae & Leaves, bark & Antidiabetic & {$[105]$} \\
\hline 83 & Hypericum perforatum & Hypericaceae & Leaves & Antidiabetic and antinociceptive & [106] \\
\hline 84 & Jatropha curcus & Euphorbiaceae & Leaves & $\begin{array}{l}\text { Ameliorate the dearrangment in lipid } \\
\text { metabolism in diabetes }\end{array}$ & [41] \\
\hline 85 & Juniiperus oxycedrus & Cupressaceae & Fruit/leaves & Hypoglycemic and antidiabetic & [107] \\
\hline 86 & Kielmeyera coriacea & Calophyllaceae & Stem/bark & $\alpha$-Amylase inhibition & [55] \\
\hline 87 & Lagenaria siceraria & Cucurbitaceae & Fruit & Antioxidant and antihyperglycemic & [63] \\
\hline 89 & Lantana camara & Verbenaceae & Leaves & Antidiabetic & [3] \\
\hline 90 & Levisticum officinale & Apiaceae & Root & $\alpha$-Glucosidase inhibition & [46] \\
\hline
\end{tabular}




\section{Continued}

\begin{tabular}{|c|c|c|c|c|c|}
\hline 91 & Ligusticum chuanxiong & Apiaceae & Aerial part & Reduce kidney damage caused by diabetes & {$[108]$} \\
\hline 92 & Liriope spicata & Liliaceae & Root & Antidiabetic & {$[3]$} \\
\hline 93 & Lithocarpus polystachyus & Fagaceae & Leaves & $\begin{array}{c}\alpha \text {-Amylase, } \alpha \text {-Glucosidase inhibition } \\
\text { and Hypoglycemic }\end{array}$ & {$[109,110]$} \\
\hline 94 & Lpomoea batatas & Convolvulaceae & Whole plant & Antihyperglycemic & {$[97]$} \\
\hline 95 & Luffa acutangula & Cucurbitaceae & Fruit & Antioxidant and antihyperglycemic & {$[63]$} \\
\hline 96 & Malmea depressa & Annonaceae & Root & $\begin{array}{l}\text { Block hepatic glucose production } \\
\text { (gluconeogenesis) }\end{array}$ & {$[111,112]$} \\
\hline 97 & Mangifera indica & Anacardiaceae & Stem bark/leaves & Antihyperglycemic & {$[14]$} \\
\hline 98 & Marrubium vulgare & Lamiaceae & Leaves & Antihyperglycemic and dyslipidemia effect & {$[113]$} \\
\hline 99 & Mimosa pudica & Fabaceae & Whole pant & Antihyperglycemic & {$[50]$} \\
\hline 100 & Momordica charantia & Cucurbitaceae & Fruit & Hypoglycemic effect, Antidiabetic effect & {$[3,114-117]$} \\
\hline 101 & Morinda cetrifolia & Rubiaceae & Leaves & Antioxidant and anticancerous activity & {$[44]$} \\
\hline 102 & Morus alba Morus nigra & Moraceae & Whole plant & $\begin{array}{c}\alpha \text {-Amylase inhibition, } \alpha \text {-Glucosidase } \\
\text { inhibition antihyperglycemic } \\
\text { and antioxidant }\end{array}$ & {$[118-120]$} \\
\hline 103 & Mucuna pruriens & Fabaceae & Seed & Hypoglycemic & {$[121]$} \\
\hline 104 & Murraya koenigii & Rutaceae & Leaves & $\begin{array}{l}\text { Antioxidant and renal pain disorder } \\
\text { among diabetes }\end{array}$ & {$[122]$} \\
\hline 106 & Nephelium lappaceumrin & Magnoliopsida & Peel & $\begin{array}{l}\alpha \text {-Amylase, } \alpha \text {-Glucosidase inhibition } \\
\text { and antihyperglycemic }\end{array}$ & {$[123,124]$} \\
\hline 107 & Nervilia plicata & Orchiaceae & Stem & Antidiabetic & {$[125]$} \\
\hline 108 & Nymphaea stellata & Nymphaeaceae & Flower & $\alpha$-Glucosidase inhibition & {$[126]$} \\
\hline 109 & Ocimum sanctum & Labiatae & Whole plant & $\begin{array}{l}\text { Antioxidant, anti cataract activity, } \\
\text { ameliorate the derangement in lipid } \\
\text { metabolism in diabetics and antidiabetic }\end{array}$ & {$[41,44,127]$} \\
\hline 110 & Olea europaea & Oleaceae & Whole plant & Antidiabetic & {$[76]$} \\
\hline 111 & Ophiopogon japonicas & Asparagaceae & Whole plant & $\begin{array}{c}\text { Hypoglycemic, antiischemic and reduce } \\
\text { insulin resistance }\end{array}$ & {$[128]$} \\
\hline 112 & $\begin{array}{l}\text { Opuntia humifusa } \\
\text { Opuntia dillenii }\end{array}$ & Cactaceae & Stem, aerial part & Hypoglycemic and hypolipidemic & {$[129,130]$} \\
\hline 113 & Opuntia streptacantha & Cactaceae & Leaves & Antihyperglycemic & {$[131]$} \\
\hline 114 & Palo fierro & Fabaceae & Seed & $\alpha$-Amylase inhibition & {$[132]$} \\
\hline 115 & Panax ginseng & Araliaceae & Root & Increase plasma insulin level & {$[12,33,133-135]$} \\
\hline 116 & Panax quinquefolius & Araliaceae & Bark & Hypoglycemic & {$[136]$} \\
\hline 117 & Parinari excelsa & Chrysobalanaceae & Bark & Antidiabetic & {$[3]$} \\
\hline 118 & Peltophorum pterocarpum & Fabaceae & Leaves/bark & $\begin{array}{l}\alpha \text {-Amylase, } \alpha \text {-Glucosidase inhibition, } \\
\text { aldose reductase inhibition, } \\
\text { antioxidant and antiglycemic. }\end{array}$ & {$[137]$} \\
\hline 119 & Phyllanthus amarus & Phyllanthaceae & Whole plant & Antidiabetic & {$[3,138]$} \\
\hline
\end{tabular}




\section{Continued}

\begin{tabular}{|c|c|c|c|c|c|}
\hline 120 & Pilea microphylla & Urticaceae & Leaves & Antihyperlipidemic and antioxidant & {$[139]$} \\
\hline 121 & Pimpinella tirupatiensis & Apiaceae & Tuberous root & $\begin{array}{l}\text { Repress oxidative stress induced } \\
\text { by diabetes }\end{array}$ & {$[140]$} \\
\hline 122 & Pine & Pinaceae & Pine bark & A-Amylase and $\alpha$-Glucosidase inhibition & {$[141]$} \\
\hline 123 & Pistacia vera & anacardiaceae & Fruits hull & A-Glucosidase inhibition & {$[46]$} \\
\hline 124 & Pongamia pinnata & Fabaceae & Seed/flower & $\begin{array}{c}\text { Antioxidant, } \beta \text {-carotene degradation, } \\
\alpha \text {-Amylase and } \alpha \text {-Glucosidase inhibition }\end{array}$ & {$[22,142]$} \\
\hline 125 & Portulaca oleracea & Portulacaceae & Seed & $\begin{array}{l}\text { Hypoglycemic, hypolipidaemic and } \\
\text { reduce insulin resistance }\end{array}$ & [143] \\
\hline 126 & Prosopis glandulosa & Fabaceae & Leaves & Increase insulin secretion & {$[144]$} \\
\hline 127 & Prunus amygdalus & Rosaceae & Seed & Antidiabetic & {$[3]$} \\
\hline 128 & Psidium guajava & Myrtaceae & Leaves & $\begin{array}{c}\text { Relief in cardiovascular complication } \\
\text { associated with diabetes and } \\
\alpha \text {-Amylase inhibition }\end{array}$ & {$[9,145]$} \\
\hline 129 & Psoralea corlifolia & Fabaceae & Leaves & Antioxidant & {$[44]$} \\
\hline 130 & $\begin{array}{l}\text { Pterocarpus santalinus, } \\
\text { Pterocarpus marsupism }\end{array}$ & Fabaceae & Bark/wood bark/leaves & $\begin{array}{c}\text { Hypoglycemic, improve hyperlipidema, } \\
\text { antihyperglycemic and prevent } \\
\text { mucosal ulceration }\end{array}$ & {$[34,146-148]$} \\
\hline 131 & Pueraria lobata & Fabaceae & Root & $\begin{array}{l}\text { Antidiabetic, } \alpha \text {-Amylase and } \\
\alpha \text {-Glucosidase inhibition }\end{array}$ & {$[56,149]$} \\
\hline 132 & Pumpkin & Cucurbitaceae & Fruit/seed & Hypoglycemic & {$[150,151]$} \\
\hline 133 & Punica granatum & Lythraceae & Fruit & Antidiabetic & {$[3]$} \\
\hline 134 & Rauwolfia serpentine & Apocyanaceae & Leaves & Hypoglycemic & {$[50]$} \\
\hline 135 & Rheum emodi wall ex. & Polygonaceae & Rhizome & $\alpha$-Glucosidase inhibition & {$[152]$} \\
\hline 136 & Rhus verniciflua & Anacardiaceae & Stem & $\alpha$-Glucosidase inhibition & {$[10]$} \\
\hline 137 & Ricinus communis & Euphorbiaceae & Root & Antidiabetic & {$[3]$} \\
\hline 138 & Ramulus cinnamomi & lauraceae & Aerial part & $\alpha$-Glucosidase inhibition & {$[56]$} \\
\hline 139 & Rosa damascene & Rosaceae & floret & $\alpha$-Glucosidase inhibition & {$[46]$} \\
\hline 140 & Rosmarinus officinalis & Lamiaceae & Aerial part & $\begin{array}{c}\text { Antidiabetic and } \alpha \text {-Glucosidase } \\
\text { inhibition }\end{array}$ & {$[46,76]$} \\
\hline 141 & Rumex patientia & Polygonaceae & Seed & $\begin{array}{l}\text { Antihyperglycemic and } \\
\text { antihyperlipidemia }\end{array}$ & {$[153]$} \\
\hline 142 & $\begin{array}{l}\text { Salacia reticulate, } \\
\text { Salacia oblonga wall }\end{array}$ & Celastraceae & Leaves/root bark & $\alpha$-Glucosidase inhibition, Antidiabetic & {$[3,154]$} \\
\hline 143 & Salvadora persica & Salvadoraceae & wood & $\alpha$-Glucosidase inhibition & {$[46]$} \\
\hline 144 & Sanguisorba minor & Rosaceae & Aerial part & $\alpha$-Glucosidase inhibition & {$[46]$} \\
\hline 145 & Sarcopoterium spinosum & Rosaceae & Root & Antidiabetic & {$[3]$} \\
\hline 146 & Selaginella tamariscina & Selaginellaceae & Aerial part & $\begin{array}{l}\text { Antihyperglycemic and } \\
\text { antihyperlipidemia }\end{array}$ & {$[155]$} \\
\hline 147 & Sechium edule & Cucurbitaceae & Fruit & Antioxidant and hyperglycemic & {$[63]$} \\
\hline 148 & Silybum marianum & Asteraceae & Whole plant & Antihyperglycemic & {$[97]$} \\
\hline
\end{tabular}




\section{Continued}

\begin{tabular}{|c|c|c|c|c|c|}
\hline 149 & Smallanthus sonchifolius & Asteraceae & Root/leaves & Hypolipidemic, antidiabetic & {$[3,156]$} \\
\hline 150 & Solanum melongena & Solanaceae & Fruit & Antioxidant and hyperglycemic & {$[63]$} \\
\hline 152 & Sorghum & Cereal crop & Grain & $\alpha$-Amylase and $\alpha$-Glucosidase inhibition & {$[158]$} \\
\hline 153 & Stevia rebaudiana & Asteraceae & Leaves & Hypoglycemic & {$[159-161]$} \\
\hline 154 & $\begin{array}{l}\text { Swertia punicea } \\
\text { Swertia chirata }\end{array}$ & Gentianaceae & Whole plant & Antidiabetic & {$[3,162]$} \\
\hline 155 & $\begin{array}{l}\text { Symplocos } \\
\text { cochinchinensis }\end{array}$ & Symplocaceae & Leaves & Antidiabetic & {$[163]$} \\
\hline 157 & Tectona grandis & Lamiaceae & Flower & $\begin{array}{l}\text { Antidiabetic, antihyerlipidemic } \\
\text { and antioxidant }\end{array}$ & {$[168]$} \\
\hline 158 & Terminalia bellerica & Cobretaceae & Fruit & $\begin{array}{l}\text { Antioxidant, } \alpha \text {-Amylase, } \alpha \text {-Glucosidase } \\
\text { inhibition, promote insulin secretion, } \\
\text { regenerate } \beta \text {-cells and antiglycation }\end{array}$ & {$[93,169]$} \\
\hline 159 & Teucrium capitatum & Lamiaceae & Whole plant & Antidiabetes & {$[76]$} \\
\hline 160 & Thymus serpyllum & Lamiaceae & Aerial part & $\alpha$-Glucosidase inhibition & {$[46]$} \\
\hline 161 & Tinospora cordifolia & Menispermaceae & Stem & $\begin{array}{l}\text { Antioxidant, anti cataract activity, } \\
\text { meliorate the derangement in lipid } \\
\text { metabolism in diabetes }\end{array}$ & {$[41,44]$} \\
\hline 163 & Tetracera scandens & Dilleniaceae & Leaves & Antihyperglycemic & {$[170]$} \\
\hline 164 & Trichosanthes cucumerina & Cucurbitaceae & Fruit & Antioxidant and Hypoglycemic & {$[63]$} \\
\hline 165 & Trigonella berythea & Fabaceae & Whole plant & Antidiabetic & {$[76]$} \\
\hline 166 & Uncaria tomentosa & Rubiaceae & Leaves/bark & Anti immune mediated diabetes & {$[171]$} \\
\hline 167 & Vaccinium arcto-staphylus & Ericaceae & fruits & $\alpha$-Glucosidase inhibition & {$[46]$} \\
\hline 168 & Vaccinium bracteatum tumb & Ericaceae & Leaves & Hypoglycemic & {$[172]$} \\
\hline 169 & Verbascum kermanensis & Scrophulariaceae & Leaves & $\alpha$-Glucosidase inhibition & {$[46]$} \\
\hline 171 & $\begin{array}{l}\text { Vernonia amygdalina, } \\
\text { Vernonia anthelmintica }\end{array}$ & Asteraceae & Aerial part/seed & $\begin{array}{l}\text { Hypoglycemic and inhibition of hepatic } \\
\text { G6pase, antidiabetic }\end{array}$ & {$[3,173]$} \\
\hline 172 & Vitis vinifera $L$. & Vitaceae & Seed/skin & $\begin{array}{l}\alpha \text {-Amylase, } \alpha \text {-Glucosidase inhibition } \\
\text { and antihyperglycemic }\end{array}$ & {$[174,175]$} \\
\hline 173 & Zataria multiflora & Lamiaceae & Aerial part & $\alpha$-Glucosidase inhibition & {$[46]$} \\
\hline 174 & Zhumeria majdae & Lamiaceae & leaves & $\alpha$-Glucosidase inhibition & {$[46]$} \\
\hline 175 & Zingiber officinale & Zingiberaceae & Root & Hypoglycemic and antioxidant & {$[176]$} \\
\hline 176 & Zizyphus spinachristi & Rhamnaceae & Leaves & $\begin{array}{l}\text { Increase insulin secretion } \\
\text { and Hypoglycemic }\end{array}$ & {$[177]$} \\
\hline 177 & Zygophyllum album & Zygophyllaceae & Leaves/root & Recovery $\beta$-cell damage and antioxidant & {$[178]$} \\
\hline
\end{tabular}


Table 2. List of medicinal herbs used in ayurveda having antidiabetic activity.

\begin{tabular}{|c|c|c|c|c|}
\hline S. No. & Vernacular name & Botanical name & Family & Parts used \\
\hline 1 & Adulsa & Adhatoda vasica & Acanthaceae & Leaves Jaswand \\
\hline 2 & Arjunsal & Terminella arjuna & Comberetaceae & Dried stem \\
\hline 3 & Aswagandha & Withania somnifera & Solanaceae & Root, leaves \\
\hline 4 & Babhul & Acacia Arabica & Leguminaceae & Gummy exudation of stem and bark \\
\hline 5 & Behda & Terminalia belleric & Combretaceae & Fruits \\
\hline 6 & Betel nut & Areca catechu & Palmitaceae & Dried ripe seeds \\
\hline 7 & Bitter gourd & Mimordica chirantia & Cucurbitaceae & Fresh green leaves \\
\hline 8 & Brahmi & Bacopa monniera & Scrophulariaceae & Aerial parts \\
\hline 9 & Chirait & Swertia chirata & Gentinaceae & Entire herbs \\
\hline 10 & Gudmar & Gymnema sylvestre & Asclepidaceae & Dried leaves \\
\hline 11 & Guggul & Commiphora mukul & Burseraceae & Oleo gum resin incision of stem bark \\
\hline 12 & Gulvel & Tinospora cardifoilia & Menispermaceae & Stem, roots \\
\hline 13 & Hirda & Terminlia chebula & Combretaceae & Fruits \\
\hline 14 & Jambuphal & Syzygium cumini & Myrtaceae & Mature fruits, dried seeds \\
\hline 15 & Jaswand & Hibiscusrosa sinensis & Malvaceae & Flower \\
\hline 16 & Kutas & Petrocarpus marsupium & Leguminaceae & Dried Juice of plant \\
\hline 17 & Nagarmotha & Cyprus rotandus & Cyperaceae & Rhizome \\
\hline 18 & Pimli & Piper longum & Piperaceae & Leaves \\
\hline 19 & Sunth & Zingiber officinalis & Zingiberaceae & Rhizome \\
\hline 20 & Tagar & Valarina wallichi & Valerianaceae & Rhizome, stolen, root \\
\hline 21 & Tulsi & Ocimum sanctum & Labiateae & Entire herbs \\
\hline 22 & Yasti & Glycyrrhiza glabra & Leguminaceae & Roots, stolen \\
\hline
\end{tabular}

patient with Type II diabetes and free from any significant side effect [34].

\section{Herbal Drugs and Its Organic Compounds for Treatment of Diabetes Mellitus}

Mordern treatments (synthetic drugs) of diabetes mellitus are focused on lowering the glucose level to normal level into blood while traditional medicines/drugs/extracts are complex in nature as well as their mechanism of action. Ayurvedic formulations, often complex with several herbal-mineral ingredients, are governed by well-described pharmacological principles of preparation, compatibility and administration.

Classic texts contain descriptions of classic formulations, traditional Ayurvedic practitioners often modify them to suit the individual constitution (prakriti), which confers genetic predisposition toward disease and therapy response, and is vital to ensure medication safety
[183]. It is very hard or laborious work to isolate and identify each and every compound found in plant extracts. However researchers find out some compounds from extract of plants and their actions are also studied. List of some plants, their isolated compounds and mechanism of action are tabled in Table 3.

\section{Need and Importance of Herbal Medicine}

As we mention above the cure of diabetes mellitus is mentioned in Ayurveda. Ayurvedic researches undertaken during the last 50 years have not been very rewarding, except for the extremely useful exercise of literary research. Further the age of synthetic drugs comes but unfortunately, after the introduction of sulfonylurea and metformin about 50 years no major lead has been obtained in the direction of proper treatment of diabetes. This is the big question mark on synthetic drugs for answering the diabetes mellitus. 
Table 3. List of compounds found in herbal plants.

\begin{tabular}{|c|c|c|c|c|c|}
\hline S. No. & Plant name (botanical) & Isolated compounds & Structure of compounds & Effects observed & Reference(s) \\
\hline 1 & Myrcia multiflora DC & Myrciacitrin I to $\mathrm{V}$ & Figure 1 & Aldose reductase Inhibitory activity & [179] \\
\hline \multirow[t]{6}{*}{2} & Stephania tetrandra S. Moore & Tetrandrine 2 '-N- $\beta$-oxide & Figure 2 & Antihyperglycemic & [180] \\
\hline & & Tetrandrine 2 '-N- $\alpha$-oxide & Figure 2 & Antihyperglycemic & [180] \\
\hline & & Tetrandrine $2-\mathrm{N}-\beta$-oxide & Figure 3 & Antihyperglycemic & {$[180]$} \\
\hline & & Fangchinoline 2'-N- $\alpha$-oxide & Figure 3 & Antihyperglycemic & {$[180]$} \\
\hline & & 2'-N-norfangchinoline & Figure 3 & Antihyperglycemic & {$[180]$} \\
\hline & & 2'-N-methyltetrandrinium chloride & Figure 3 & Antihyperglycemic & [180] \\
\hline 3 & Syzygium malaccense (L.) & Casuarine 6 -O- $\alpha$-glucoside & Figure 4 & $\alpha$-Glucosidase inhibition & [181] \\
\hline \multirow[t]{3}{*}{4} & Tecoma stans (L.) & Tecomine & Figure 5 & Insulin-mimic & [182] \\
\hline & & $5 \beta$-hydroxyskitanthine & Figure 5 & Insulin-mimic & [182] \\
\hline & & Boschniakine & Figure 5 & Insulin-mimic & {$[182]$} \\
\hline
\end{tabular}<smiles>Cc1c(O)c(C)c2c(c1O)C(=O)C[C@@H](c1cc(O)ccc1O[C@H]1OC(CO)[C@@H](O)[C@H](O)[C@H]1O)O2</smiles>

Myrciacitrin-III<smiles>Cc1c(O)c2c(c(C)c1O[C@H]1O[C@H](COC(=O)c3ccc(O)cc3)[C@@H](O)[C@H](O)[C@H]1O)O[C@H](c1cc(O)ccc1O)CC2=O</smiles>

Myrciacitrin-V<smiles>Cc1c(O)c2c(c(C)c1O[C@H]1O[C@H](COC(=O)/C=C/c3ccc(O)cc3)[C@@H](O)[C@H](O)[C@H]1O)O[C@H](c1cc(O)ccc1O)CC2=O</smiles>

Myrciacitrin-IV<smiles>COc1ccc(O)c([C@H]2CC(=O)c3c(C)c4c(c(C)c3O)O[C@@H](O4)[C@H](O)[C@H]2O)c1</smiles>

Myrciacitrin-II

Figure 1. Representing structure of Myrciacitrin I to V. These molecules were isolateted from Myrcia multiflora DC. (Myrtaceae). 


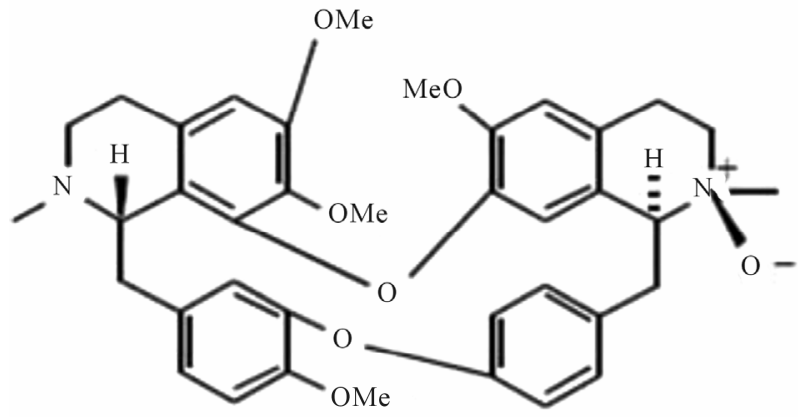

Tetrandrine2'-N- $\beta$-oxide

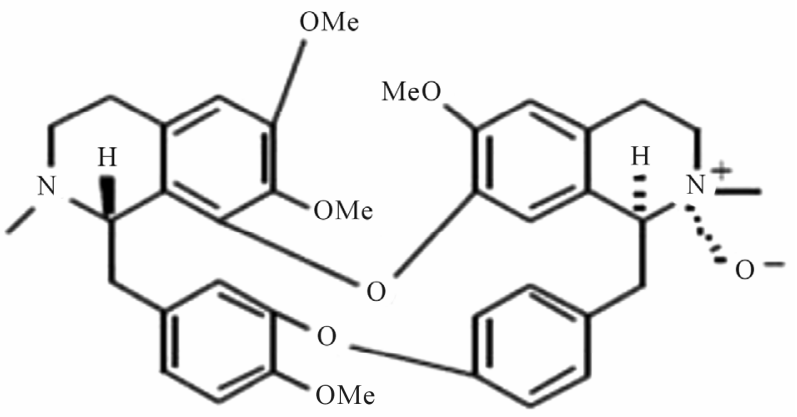

Tetrandrine 2'-N- $\alpha$-oxide

Figure 2. Representing structure of six bis-benzylisoquinoline-type alkaloids. These molecules were separated from Stephania tetrandra S. Moore (Menispermaceae).

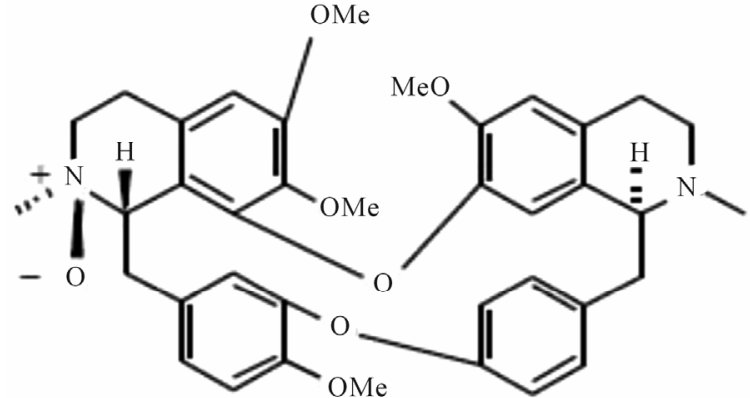

Tetrandrine2-N- $\beta$-oxide<smiles></smiles>

2'-N-norfangchinoline

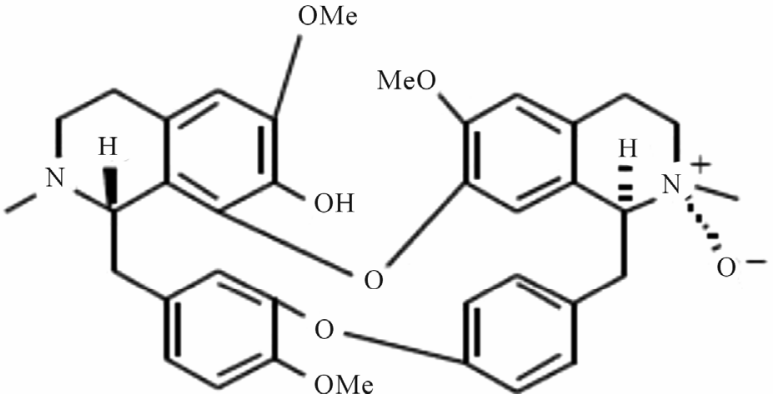

Fangchioline 2'-N- $\alpha$-oxide

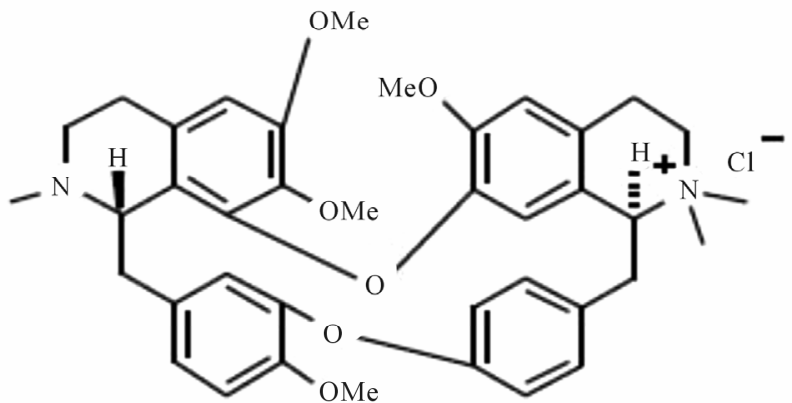

2'-N-methyltetrandrinium chloride

Figure 3. Representing structure of six bis-benzylisoquinoline-type alkaloids. These molecules were separated from Stephania tetrandra S. Moore (Menispermaceae).

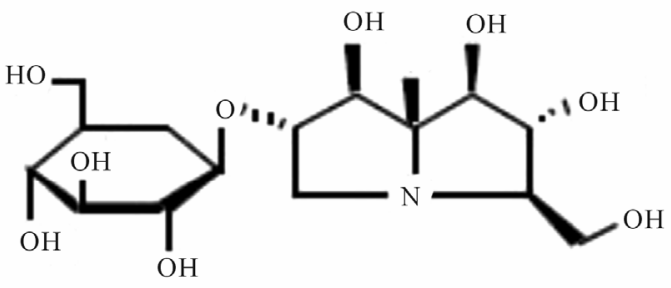

Casuarine 6-O- $\alpha-$ glucoside

Figure 4. Representing structure of casuarine 6-O- $\alpha$-glucoside alkaloids. This molecule was isolated from Syzygium malaccense (L.) Merrill \& L. M. Perry (Myrtaceae). 


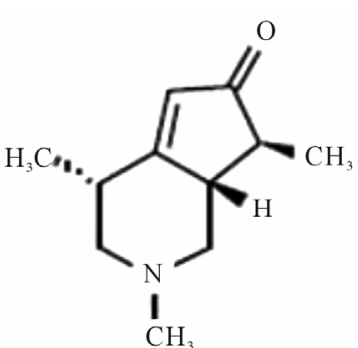

Tecomine

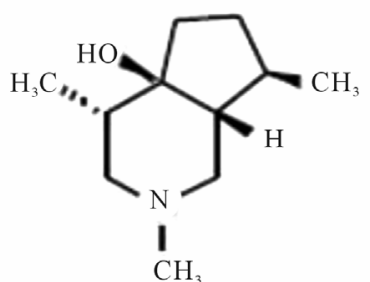

$5 \beta$-hydroxyskitanthine

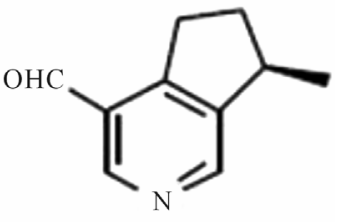

Boschiakine

Figure 5. Representing structure of molecules isolated from Tecoma stans (L.) Juss. Ex kunth (Bignoniaceae).

Plant extract or different folk plants preparations are being prescribed by the traditional practitioners and also accepted by the users for diabetes and other diseases in many countries especially in third world countries. Therefore, a proper scientific evaluation and searching plants by pharmacological test followed by chemical investigation is potentially necessary.

The plants drugs or extract have the marvelous efficacy to curing the diabetes and its complication without having any side effects. At present there are several Chinese traditional formulated drugs available in market but the right answer for diabetes mellitus is awaited.

According to the WHO about $65 \%-80 \%$ of world population in developing counties depends on all intents and purposes on plant for their primary health care due to scarcity and lack of access to modern medicine [35]. Historically all medicinal preparation were derive from plants, whether in the simple form of plant parts or in the more complex form of crude extract mixture etc. The primary benefits of using plant-derived medicines are that they are relatively safer than synthetic alternative drug [36]. Use of ethanobotanical information in medicinal plant research has gain considerable attention in segment of the scientific community [37].

As we know that India has ancient heritage of traditional medicine. In India where $75 \%$ population belong from the remote area and more than $50 \%$ of people survives below the poverty line enthusiastically use plants for the treating the diseases. During the last few decease there has been an increasing interest in the study of medicinal plant and their traditional use in different part of India.

In the recent years numbers of reports on the use of plants in traditional healing by either tribal people or indigenous communities of India is increasing [38]. Muniappan Ayyanar et al. 2011 [39] conducted a study in Tirunelveli hills of Western Ghats in India and found that Costus speciosus smith (Costaceae), Gymnema sylvestre Linn (Apocynaceae) are used for treating diabetes mellitus. In this review we have concluded that plants are best sources for developing the alternative drug to curing diabetes mellitus, which has no side effects and low cost by which it is simple to a common man to be treated. There is a need of searching our traditional medicines. Some of ethanobotanical plants are listed here in Tables $\mathbf{1}$ and $\mathbf{2}$ which has antidiabetic properties.

\section{Conclusion}

All drugs which we have discussed in this review have a significant in their mode of action and therapy of diabetes mellitus, in contrast of plants bioactive phyto-molecules are less known about their mode of action but there is no doubt about the role of plants to treating diabetes.

It is also important to screening the world's plant diversity extensively for more and specific bioactive phytomolecules which are helpful in treating diabetes mellitus. On the other hand the traditional formulation of antidiabetic drugs must be researched and re-standardized by using new techniques and methods for managing the diabetes mellitus. Furthermore these drugs will be accessible to the people who are unable to purchase the costly synthetic drugs. Hence herbal drugs may be an emerging alternative of synthetic drugs to curing diabetes mellitus.

\section{REFERENCES}

[1] K. A. Wadkar, C. S. Magdum, S. S. Patil and N. S. Naikwade, "Anti-Diabetic Potential and Indian Medicinal Plants," Journal of Herbal Medicine and Toxicology, Vol. 2, No. 1, 2008, pp. 45-50.

[2] S. Kumar, S. Narwal, V. Kumar and O. Prakash, " $\alpha$ Glucosidase Inhibitors from Plants: A Natural Approach to Treat Diabetes," Pharmacogonsy Review, Vol. 5, No. 9, 2011, pp. 19-29. doi:10.4103/0973-7847.79096

[3] M. U. Rao, M. Sreenivasulu, B. Chengaiah, K. J. Reddy and C. M. Chetty, "Herbal Medicines for Diabetes Mellitus: A Review," International Journal of PharmTech Research, Vol. 2, No. 3, 2010, pp. 1883-1892.

[4] J.-H. Park, S. Ko and H. Park, "Toward the Virtual Screening of $\alpha$-Glucosidase Inhibitors with the Homology-Modeled Protein Structure," Bulletin of the Korean Chemical Society, Vol. 29, No. 5, 2008, pp. 921-927. doi:10.5012/bkcs.2008.29.5.921

[5] H. Park, K. Y. Hwang, Y. H. Kim, K. H. Oh, J. Y. Lee and K. Kim, "Discovery and Biological Evaluation of 
Novel $\alpha$-Glucosidase Inhibitors with in Vivo Antidiabetic Effect," Bioorganic \& Medicinal Chemistry Letters, Vol. 18, 2008, pp. 3711-3715. doi:10.1016/j.bmcl.2008.05.056

[6] A. Klip, T. Ramlal, P. J. Bilan, G. D. Cartee, E. A. Gulve and J. O. Holloszy, "Recruitment of GLUT-4 Glucose Transporters by Insulin in Diabetic Rat Skeletal Muscle," Biochemical and Biophysical Research Communications, Vol. 172, No. 2, 1990, pp. 728-736. doi:10.1016/0006-291X(90)90735-6

[7] A. R. Saltiel and C. R. Kahn, "Insulin Signalling and the Regulation of Glucose and Lipid Metabolism," Nature, Vol. 414, No. 6865, 2001, pp. 799-806. doi:10.1038/414799a

[8] M. J. Salgueiro, N. Krebs, M. B. Zubillaga, R. Weill, E. Postaire, A. E. Lysionek, R. A. Caro, T. De Paoli, A. Hager and J. Boccio, "Zinc and Diabetes Mellitus Is There a Need of Zinc Supplementation in Diabetes Mellitus Patients?" Biological Trace Element Research, Vol. 81, No. 3, 2001, pp. 215-228. doi:10.1385/BTER:81:3:215

[9] A. Singh and T. Marar, "Inhibitory Effect of Extracts of Syzygium cumini and Psidium guajava on Glycosidases," Journal of Cell and Tissue Research, Vol. 11, No. 1, 2011, pp. 2535-2539.

[10] J.-S. Kim, J. F. Yang and M.-J. Kim, “Alpha Glucosidase Inhibitory Effect, Anti-Microbial Activity and UPLC Analysis of Rhus verniciflua under Various Extract Conditions," Journal of Medicinal Plants Research, Vol. 5, No. 5, 2011, pp. 778-783.

[11] M.-M. Si, J.-S. Lou, C.-X. Zhou, J.-N. Shen, H.-H. Wu, B. Yang, Q.-J. He and H.-S. Wu, "Insulin Releasing and Alpha-Glucosidase Inhibitory Activity of Ethyl Acetate Fraction of Acorus calamus in Vitro and in Vivo," Journal of Ethnopharmacology, Vol. 128, No. 1, 2010, pp. 154-159. doi:10.1016/j.jep.2009.12.044

[12] W.-K. Lee, S.-T. Kao, I.-M. Liu and J.-T. Cheng, "Increase of Insulin Secretion by Ginsenoside RH2 to Lower Plasma Glucose in Wistar Rats," Clinical and Experimental Pharmacology and Physiology, Vol. 33, No. 1-2, 2006, pp. 27-32. doi:10.1111/j.1440-1681.2006.04319.x

[13] C. D. Rosely, C. Melazzo, S. C. A. da Luz, A. Fiappi, D. Prestes, A. F. da Silveira and M. Cecim, "Syzygium cumini and the Regeneration of Insulin Positive Cells from the Pancreatic Duct," Brazilian Journal of Veterinary Research and Animal Sciences, Vol. 41, No. 4, 2004, pp. 236-239.

[14] A. Bhowmik, L. Ali Khan, M. Akhter and B. Rokeya, "Studies on the Antidiabetic Effects of Mangifera indica Stem-Barks and Leaves on Nondiabetic, Type 1 and Type 2 Diabetic Model Rats," Bangladesh Journal of Pharmacology, Vol. 4, No. 2, 2009, pp. 110-114. doi:10.3329/bjp.v4i2.2488

[15] P. P. Reddy, A. K. Tiwari, R. R. Rao, K. Madhusudhana, V. R. S. Rao, A. Z. Ali, K. S. Babu and J. M. Rao, "New Labdane Diterpenes as Intestinal $\alpha$-Glucosidase Inhibitor from Antihyperglycemic Extract of Hedychium spicatum ( Ham. Ex Smith) Rhizomes," Bioorganic \& Medicinal Chemistry Letters, Vol. 19, No. 9, 2009, pp. 2562-2565. doi:10.1016/j.bmcl.2009.03.045
[16] M. Gayathri and K. Kannabiran, "Antidiabetic and Ameliorative Potential of Ficus bengalensis Bark Extract in Streptozotocin Induced Diabetic Rats," Indian Journal of Clinical Biochemistry, Vol, 23, No, 4, 2008, pp. 394-400. doi:10.1007/s12291-008-0087-2

[17] R. Kuriyan, R. Rajendran, G. Bantwal and A. V. Kurpad, "Effect of Supplementation of Coccinia cordifolia Extract on Newly Detected Diabetic Patients," Diabetes Care, Vol. 31, No. 2, 2008, pp. 216-220. doi:10.2337/dc07-1591

[18] M. S. Akhtar and A. Q. Qureshi, "Phytopharmacological Evaluation of Ficus glomerata, Roxb. Fruit for Hypoglycemic Activity in Normal and Diabetic Rabbits," Pakistan Journal of Pharmaceutical Sciences, Vol. 1, No. 2, 1988, pp. 87-96.

[19] H. Kirana, S. S. Agrawal and B. P. Srinivasan, "Aqueous Extract of Ficus religiosa Linn. Reduse Oxidative Stress in Experimentally Induced Type 2 Diabetic Rats," Indian Journal of Experimental Biology, Vol. 47, 2009, pp. 822826.

[20] B. V. Shetty, A. Arjuman, A. Jorapur, R. Samanth, S. K. Yadav, V. N. Anna Deephy Tharian, S. K. and G. M. Rao, "Effect of Extract of Benincasa hispida on Oxidative Stress in Rats with Indomethacin Induced Gastric U1cers," Indian Journal of Pharmacology, Vol. 52, No. 2, 2008, pp. 178-182.

[21] S. A. Rao, P. V. Srinivas, A. K. Tiwari, U. M. S. Vanka, R. V. Subba Rao, K. R. Dasari and Ma. J. Rao, "Isolation, Characterization and Chemobiological Quantification of Alpha-Glucosidase Enzyme Inhibitory and Free Radical Scavenging Constituents from Derris scandens Benth," Journal of Chromatography B, Vol. 855, No. 2, 2011, pp. 166-172.

[22] R. Punitha, K. Vasudevan and S. Manoharan, "Effect of Pongamia pinnata Flower on Blood Glucose and Oxidative Stress in Alloxan Induced Diabetic Rats," Indian Journal of Pharmacology, Vol. 38, No. 1, 2006, pp. 6263. doi:10.4103/0253-7613.19858

[23] S. Venkateswaran and L. Pari, "Effect of Coccinia indica Leaves on Antioxidant Status in Streptozotocin-Induced Diabetic Rats," Journal of Ethnopharmacology, Vol. 84, No. 2-3, 2003, pp. 163-168. doi:10.1016/S0378-8741(02)00294-5

[24] S. Venkateswaran and L. Pari, "Effect of Coccinia indica Leaf Extract on Plasma Antioxidants in Streptozotocin Induced Experimental Diabetes in Rats," Phytotherapy Research, Vol. 17, No. 6, 2003, pp. 605-608. doi:10.1002/ptr.1195

[25] S. Venkateswaran, L. Pari, L. Suguna and G. Chandrakasan, "Modulatory Effect of Coccinia indica on Aortic Collagen in Streptozotocin-Induced Diabetic Rats," Clinical and Experimental Pharmacology and Physiology, Vol. 30, No. 3, 2003, pp. 157-163.

doi:10.1046/j.1440-1681.2003.03812.x

[26] P. K. Mukherjee, P. Venkatesh and S. Ponnusankar, "Ethnopharmacology and Integrative Medicine-Let the History Tell the Future," Journal of Ayurveda \& Integrative Medicine, Vol. 1, No. 2, 2010, pp. 100-109. doi:10.4103/0975-9476.65077

[27] R. H. Singh, "Exploring Issues in the Development of 
Ayurvedic Research Methodology," Journal of Ayurveda \& Integrative Medicine, Vol. 1, No. 2, 2010, pp. 91-95. doi:10.4103/0975-9476.65067

[28] A. K.Gupta, "Quality Standard of Indian Medicinal Plants," Vol. 1, International Center for Materials Research, New Delhi, 1986, pp. 168-173.

[29] J. K. Grover, S. Yadav and V. Vats, "Medicinal Plants of India with Anti-Diabetic Potential," Journal of Ethnopharmacology, Vol. 81, No. 1, 2002, pp. 81-100. doi:10.1016/S0378-8741(02)00059-4

[30] R. Mishra, M. Shuaib, S. and P. S. Mishra, "Areview on Herbal Antidiabetic Drugs," Journal of Applied Pharmaceutical Science, Vol. 1, No. 6, 2011, pp. 235-237.

[31] M. Modak, P. Dixit, J. Londhe, S. Ghaskadbi and T. P. A. Devasagayam, "Indian Herbs and Herbal Drugs Used for the Treatment of Diabetes," Journal of Clinical Biochemistry and Nutrition, Vol. 40, 2007, 163-173. doi:10.3164/jcbn. 40.163

[32] W. D. Winters, Y. S. Huo and D.-L. Yao, "Inhibition of the Progression of Type 2 Diabetes in the C57BL/6J Mouse Model by an Ant-Diabetes Herbl Frmula," Phytotherapy Research, Vol. 17, No. 6, 2003, pp. 591-598. doi: $10.1002 /$ ptr. 1079

[33] I. Kimura, N. Nakashima, Y. Sugihara, F.-J. Chen and M. Kimura, "The Antihyperglycemic Blend Effect of Traditional Chinese Byakko-ka-Ninjin on Alloxan a Diabetic KK-CA ${ }^{\mathrm{y}}$ Mice," Phytotherapy Research, Vol. 13, No. 6, 1999, pp. 484-488.

doi:10.1002/(SICI)1099-1573(199909)13:6<484::AID-PT R485>3.0.CO;2-X

[34] ICMR Study Group, "Efficacy of Vijayasar (Pterocarpus marsupium) in the Treatment of Newly Diagnosed Patients with Type 2 Diabetes Mellitus: A Flexible Dose Double-Nlind Multicenter Randomized Controlled Trial," Diabetologia Crotica, Vol. 34, No. 1, 2005, pp. 13-20.

[35] J. B. Calixto, "Twenty-five Years of Research on Medicinal Plants in Latin America: A Personal View," Journal of Ethnopharmacology, Vol. 100, No. 1-2, 2005, pp. 131-134. doi:10.1016/j.jep.2005.06.004

[36] M. M. Iwu, A. R. Duncan and C. O. Okunji, "New Antimicrobials of Plant Origin," In: J. Janick, Ed., Perspectives in New Crops and New Uses, ASHS Press, Alexandria, 1999, pp. 457-462.

[37] M. Heinrich and S. Gibbons, "Ethnopharmacology in Drug Discovery: An Analysis of Its Role and Potential Contribution," Journal of Pharmacy and Pharmacology, Vol. 53, No. 4, 2001, pp. 425-432. doi: $10.1211 / 0022357011775712$

[38] B. Upadhyay, Parveen, A. K. Dhaker and A. Kumar, "Ethnomedicinal and Ethnopharmaco-Statistical Studies of Eastern Rajasthan," Indian Journal of Ethnopharmacology, Vol. 129, No. 1, 2010, pp. 64-86. doi:10.1016/j.jep.2010.02.026

[39] M. Ayyanar and S. Ignacimuthu, "Ethnobotanical Survey of Medicinal Plants Commonly Used by Kani Tribals in Tirunelveli Hills of Western Ghats, India," Journal of Ethnopharmacology, Vol. 134, No. 3, 2011, pp. 851-864. doi:10.1016/j.jep.2011.01.029
[40] M. H. Eshrat, "Effect of Coccinia indica (L.) and Abroma augusta (L.) on Glycemia, Lipid Profile and on Indicators of End-Organ Damage in Streptozotocin Induced Diabetic Rats," Indian Journal of Clinical Biochemistry, Vol. 18, No. 2, 2003, pp. 54-63. doi:10.1007/BF02867368

[41] R. N. Patil, R. Y. Patil, B. Ahirwar and D. Ahirwar, "Evaluation of Antidiabetic and Related Actions of Some Indian Medicinal Plants in Diabetic Rats," Asian Pacific Journal of Tropical Medicine, Vol. 4, No. 1, 2011, pp. 20-23. doi:10.1016/S1995-7645(11)60025-4

[42] J. C. Ikewuchi, E. N. Onyeike, A. A. Uwakwe and C. C. Ikewuchi, "Effect of Aqueous Extract of the Leaves of Acalypha wilkesiana Godseffiana' Muell Arg (Euphorbiaceae) on the Hematology, Plasma Biochemistry and Ocular Indices of Oxidative Stress in Alloxan Induced Diabetic Rats," Journal of Ethnopharmacology, Vol. 137, No. 3, 2011, pp. 1415-1424. doi:10.1016/j.jep.2011.08.015

[43] E. Apostolidis, L. Li, C. Lee and N. P. Seeram, "In Vitro Evaluation of Phenolic-Enriched Maple Syrup Extracts for Inhibition of Carbohydrate Hydrolyzing Enzymes Relevant to Type 2 Diabetes Management," Journal of Functional Foods, Vol. 3, No. 2, 2011, pp. 100-106. doi:10.1016/j.jff.2011.03.003

[44] R. N. Gacche and N. A. Dhole, "Profile of Aldosereductase Inhibition, Anti-Cataract and Free Radical Scavenging Activity of Selected Medicinal Plants: An Attempt to Standardize the Botanicals for Amelioration of Diabetes Complications," Food and Chemical Toxicology, Vol. 49, No. 8, 2011, pp. 1806-1813. doi:10.1016/j.fct.2011.04.032

[45] S. O. Oyedemi, E. A. Adewusi, O. A. Aiyegoro and D. A. Akinpelu, "Antidiabetic and Haematological Effect of Aqueous Extract of Stem Bark of Afzelia africana (Smith) on Streptozotocin-Induced Diabetic Wistar Rats," Asian Pacific Journal of Tropical Biomedicine, Vol. 1, No. 5, 2011, pp. 353-358. doi:10.1016/S2221-1691(11)60079-8

[46] A. Gholamho, H. Fallah, F. Sharifi-Far and M. Mirtajaddini, "The Inhibitory Effect of Some Iraian Plant Extracts on the Alpha Glucosidase," Iranian Journal of Basic Medical Sciences, Vol. 11, No. 1, 2008, pp. 1-9. doi:10.1002/ptr.719

[47] A. Okyar, A. Can, N. Akev, G. Baktir and N. Sutlupinar, "Effect of Aloe Vera Leaves on Blood Glucose Level in Type I and Type II Diabetic Rat Models," Phytotherapy Research, Vol. 15, 2001, pp. 157-161.

[48] B. S. Ashok Kumar, K. Lakshman, R. Nandeesh, P. A. Arun Kumar, B. Manoj, V. Kumar and D. S. Shekar, "In Vitro Alpha-Amylase Inhibition and in Vivo Antioxidant Potential of Amaranthus spinosus in Alloxan-Induced Oxidative Stress in Diabetic Rats," Saudi Journal of Biological Sciences, Vol. 18, No. 1, 2011, pp. 1-5. doi:10.1016/j.sjbs.2010.08.002

[49] K. Girija, K. Lakshman, C. Udaya, G. S. Sachi and T. Divya, "Anti-Diabetic and Anti-Cholesterolemic Activity of Methanol Extracts of Three Species of Amaranthus," Asian Pacific Journal of Tropical Biomedicine, Vol. 1, No. 2, 2011, pp. 133-138. doi:10.1016/S2221-1691(11)60011-7

[50] J. Manosroi, Z. Z. Moses, W. Manosroi and A. Manosroi, 
"Hypoglycemic Activity of Thai Medicinal Plants Selected from the Thai/Lanna Medicinal Recipe Database MANOSROI II," Journal of Ethnopharmacology, Vol. 138, No. 1, 2011, pp. 92-98.

doi:10.1016/j.jep.2011.08.049

[51] X.-Z. Hu, X.-H. Xing, Z.-M. Zhang, R.-Q. Wu, Q. B. Guo, S. W. Cui and Q. Wang, "Antioxidant Effects of Artemis sphaerocephala Krasch. Gum, on Streptozotocin-Induced Type 2 Diabetic Rats," Food Hydrocolloids, Vol. 25, No. 2, 2011, pp. 207-213. doi:10.1016/i.foodhyd.2009.12.006

[52] S. W. Eisenman, A. Poulev, L. Struwe, I. Raskin and D. M. Ribnicky, "Qualitative Variation of Anti-Diabetic Compounds in Different Tarragon (Artemisia dracunculus L.) Cytotypes," Fitoterapia, Vol. 82, No. 7, 2011, pp. 1062-1074. doi:10.1016/i.fitote.2011.07.003

[53] N. Hamza, B. Berke, C. Cheze, R. Le Garrec, R. Lassalle, A.-N. Agli, P. Robinson, H. Gin and N. Moore, "Treatment of High Fat Diet Induced Type 2 Diabetes in C57BL/6J Mice by Two Medicinal Plants Used in Traditional Treatment of Diabetes in the East of Algeria," Journal of Ethnopharmacology, Vol. 133, No. 2, 2011, pp. 931-933. doi:10.1016/j.jep.2010.11.019

[54] F. Nwosu, J. Morris, V. A. Lund, D. Stewart, H. A. Ross and G. J. McDougall, "Anti-Proliferative and Potential Anti-Diabetic Effects of Phenolic-Rich Extracts from Edible Marine Algae," Food Chemistry, Vol. 126, No. 3, 2011, pp. 1006-1012. doi:10.1016/j.foodchem.2010.11.111

[55] E. M. Silva, A. Valencia, M. F. Grossi-de-Sá, T. L. Rocha, É. Freire, J. E. de Paula and L. S. Espindola, "Inhibitory Action of Cerrado Plants against Mammalian and Insect $\alpha$-Amylases," Pesticide Biochemistry and Physiology, Vol. 95, No. 3, 2009, pp. 141-146. doi:10.1016/j.pestbp.2009.08.003

[56] K. He, X. G. Li, X. Chen, X. L. Ye, J. Huang, Y. N. Jin, P. P. Li, Y. F. Deng, Q. Jin, Q. Shi and H. J. Shu, "Evaluation of Antidiabetic Potential of Selected Traditional Chinese Medicines in STZ-Induced Diabetic Mice," Journal of Ethnopharmacology, Vol. 137, No. 3, 2011, pp. 11351142. doi:10.1016/j.jep.2011.07.033

[57] A. B. Shori and A. S. Baba, "Antioxidant Activity and Inhibition of Key Enzymes Linked to Type-2 Diabetes and Hypertension by Azadirachta indica-Yogurt," Journal of Saudi Chemical Society, 2013 (in press).

[58] K. Biswas, I. Chattopadhyay, R. K. Banerjee and U. Bandyopadhyay, "Biological Activities and Medicinal Poperties of Neem (Azadirachta indica)," Current Science, Vol. 82, No. 11, 2002, pp. 1336-1345.

[59] P. Mangala Gowri, A. K. Tiwari, A. Z. Ali and J. M. Rao, "Inhibition of $\alpha$-Glucosidase and Amylase by Bartogenic Acid Isolated from Barringtonia racemosa Roxb. Seeds," Phytotherapy Research, Vol. 21, No. 8, 2007, pp. 796799. doi: $10.1002 / \mathrm{ptr} .2176$

[60] K. R. Patil, C. R. Patil, R. B. Jadhav, V. K. Mahajan, P. R. Patil and P. S. Gaikwad, "Anti-Arthritic Activity of Bartogenic Acid Isolated from Fruits of Barringtonia racemosa Roxb. (Lecythidaceae)," Evidence-Based Complementary and Alternative Medicine, Vol. 2011, 2011, pp. 1-7.

[61] C. M. Wu, Y. Li, Y. Chen, X. Y. Lao, L. H/ Sheng, R. J. Dai, W. W. Meng and Y. L. Deng, "Hypoglycemic Effect of Belamcanda chinensis Leaf Extract in Normal and STZ-Induced Diabetic Rats and Its Potential Active Fraction," Phytomedicine, Vol. 18, No. 4, 2011, pp. 292-297. doi:10.1016/j.phymed.2010.07.005

[62] Y. Chen, C. -M. Wu, R.-J. Dai, L. Li, Y.-H. Yu, Y. Li, W.-W. Meng, L. Zhang, Y. Q. Zhang and Y.-L. Deng, "Combination of HPLC Chromatogram and Hypoglycemic Effect Identifies Isoflavones as the Principal Active Fraction of Belamcanda chinensis Leaf Extract in Diabetes Treatment," Journal of Chromatography B, Vol. 879, No. 5-6, 2011, pp. 371-378. doi:10.1016/i.jchromb.2010.12.022

[63] A. K. Tiwari, K. S. Reddy, J. Radhakrishnan, D. A. Kumar, A. Zehra, S. B. Agawane and K. Madhusudana, "Influence of Antioxidant Rich Fresh Vegetable Juices on Starch Induced Postprandial Hyperglycemia in Rats," Food \& Function, Vol. 2, No. 9, 2011, pp. 521-528. doi:10.1039/c1fo10093a

[64] N. Meliani, M. El Amine Dib, H. Allali and B. Tabti, "Hypoglycemic Effect of Berberis vulgaris L. in Normal and Streptozotocin-Induced Diabetic Rats," Asian Pacific Journal of Tropical Biomedicine, Vol. 1, No. 6, 2011, pp. 468-471. doi:10.1016/S2221-1691(11)60102-0

[65] T. Thirumalai, S. V. Therasa, E. K. Elumalai and E. David, "Hypoglycemic Effect of Brassica juncea (Seeds) on Streptozotocin Induced Diabetic Male Albino Rat," Asian Pacific Journal of Tropical Biomedicine, Vol. 1, No. 4, 2011, pp. 323-325. doi:10.1016/S2221-1691(11)60052-X

[66] R. A. Adisa, M. I. Choudhary and O. O. Olorunsogo, "Hypoglycemic Activity of Buchholzia coriacea (Capparaceae) Seeds in Streptozotocin-Induced Diabetic Rats and Mice," Experimental and Toxicologic Pathology, Vol. 63, No. 7-8, , 2011, pp. 619-625. doi:10.1016/j.etp.2010.05.002

[67] C. F. B. Vasconcelos, H. M. L. Maranhão, T. M. Batista, E. M. Carneiro, F. Ferreira, J. Costa, L. A. L. Soares, M. D. C. Sá, T. P. Souza and A. G. Wanderley, "Hypoglycemic Activity and Molecular Mechanisms of Caesalpinia ferrea Martius Bark Extract on Streptozotocin-Induced Diabetes in Wistar Rats," Journal of Ethnopharmacology, Vol. 137, No. 3, 2011, pp. 1533-1541. doi:10.1016/j.jep.2011.08.059

[68] Y. F. Wang, S. R. Huang, S. H. Shao, L. S. Qian and P. $\mathrm{Xu}$, "Studies on Bioactivities of Tea (Camellia sinensis L.) Fruit Peel Extracts: Antioxidant Activity and Inhibitory Potential against $\alpha$-Glucosidase and $\alpha$-Amylase in Vitro," Industrial Crops and Products, Vol. 37, No. 1, 2012, pp. 520-526. doi:10.1016/j.indcrop.2011.07.031

[69] Md. S. Islam, "Effects of the Aqueous Extract of White Tea (Camellia sinensis) in a Streptozotocin-Induced Diabetes Model of Rats," Phytomedicine, Vol. 19, No. 1, 2011, pp. 25-31. doi:10.1016/j.phymed.2011.06.025

[70] B. Sharma, R. Salunke, C. Balomajumder, S. Daniel and P. Roy, "Anti-Diabetic Potential of Alkaloid Rich Fraction from Capparis decidua on Diabetic Mice," Journal 
of Ethnopharmacology, Vol. 127, No. 2, 2010, pp. 457462. doi:10.1016/j.jep.2009.10.013

[71] M. Juan-Badaturuge, S. Habtemariam and M. J. K. Thomas, "Antioxidant Compounds from a South Asian Beverage and Medicinal Plant, Cassia auriculata," Food Chemistry, Vol. 125, No. 1, 2011, pp. 221-225. doi:10.1016/j.foodchem.2010.08.065

[72] S. C. Ohadoma and H. U. Michael, "Effects of Co-Administration of Methanol Leaf Extract of Catharanthus roseus on the Hypoglycemic Activity of Metformin and Glibenclamide in Rats," Asian Pacific Journal of Tropical Medicine, Vol. 4, No. 6, 2011, pp. 475-477. doi:10.1016/S1995-7645(11)60129-6

[73] M. Sefi, H. Fetoui, N. Lachkar, A. Tahraoui, B. Lyoussi, T. Boudawara and N. Zeghal, "Centaurium erythrea (Gentianaceae) Leaf Extract Alleviates StreptozotocinInduced Oxidative Stress and $\beta$-Cell Damage in Rat Pancreas," Journal of Ethnopharmacology, Vol. 135, No. 2, 2011, pp. 243-250. doi:10.1016/j.jep.2011.02.029

[74] S. Sancheti, S. Sancheti and S.-Y. Seo, "Chaenomeles sinensis: APotent $\alpha$ and $\beta$-Glucosidase Inhibitor," American Journal of Pharmacology and Toxicology, Vol. 4, No. 1, 2009, pp. 8-11. doi:10.3844/ajptsp.2009.8.11

[75] J. Gorelick, A. Kitron, S. Pen, T. Rosenzweig, Z. Madar, "Anti-Diabetic Activity of Chiliadenus iphionoides," Journal of Ethnopharmacology, Vol. 137, No. 3, 2011, pp. 1245-1249. doi:10.1016/j.jep.2011.07.051

[76] M. S. Ali-Shtayeh, R. M. Jamous and R. M. Jamous, "Complementary and Alternative Medicine Use amongst Palestinian Diabetic Patients," Complementary Therapies in Clinical Practice, Vol. 18, No. 1, 2012, pp. 16-21. doi:10.1016/j.ctcp.2011.09.001

[77] G. Salil, K. G. Nevin and T. Rajamohan, "Arginine Rich Coconut Kernel Protein Modulates Diabetes in Alloxan Treated Rats," Chemico-Biological Interactions, Vol. 189, No. 1-2, 2011, pp. 107-111. doi:10.1016/j.cbi.2010.10.015

[78] Y. Narita and K. Inouye, "Inhibitory Effects of Chlorogenic Acids from Green Coffee Beans and Cinnamate Derivatives on the Activity of Porcine Pancreas $\alpha$-Amylase Isozyme I," Food Chemistry, Vol. 127, No. 4, 2011, pp. 1532-1539. doi:10.1016/j.foodchem.2011.02.013

[79] U. Zottich, M. Da Cunha, A. O. Carvalho, G. B. Dias, N. C. M. Silva, I. S. Santos, V. V. do Nacimento, E. C. Miguel, O. L. T. Machado and V. M. Gomes, "Purification, Biochemical Characterization and Antifungal Activity of a New Lipid Transfer Protein (LTP) from Coffea canephoraseeds with $\alpha$-Amylase Inhibitor Properties," Biochimica et Biophysica Acta (BBA)-General Subjects, Vol. 1810, No. 4, 2011, pp. 375-383. doi:10.1016/j.bbagen.2010.12.002

[80] A. Chika and S. O. Bello, "Antihyperglycemic Activity of Aqueous Leaf Extract of Combretum micranthum (Combretaceae) in Normal and Alloxan-Induced Diabetic Rats," Journal of Ethnopharmacology, Vol. 129, No. 1, 2010, pp. 34-37. doi:10.1016/j.jep.2010.02.008

[81] R. Bellamkonda, K. Rasineni, S. R. Singareddy, R. B. Kasetti, R. Pasurla, A. R. Chippada and S. Desireddy, "Antihyperglycemic and Antioxidant Activities of Alcoholic Extract of Commiphora mukul Gum Resin in Strep- tozotocin Induced Diabetic Rats," Pathophysiology, Vol. 18, No. 4, 2011, pp. 255-261. doi:10.1016/j.pathophys.2010.10.002

[82] A. Aissaoui, S. Zizi, Z. H. Israili and B. Lyoussi, "Hypoglycemic and Hypolipidemic Effects of Coriandrum sativum L. in Meriones Shawi Rats," Journal of Ethnopharmacology, Vol. 137, No. 1, 2011, pp. 652-661. doi:10.1016/j.jep.2011.06.019

[83] D. Kim, K. K. Park, S. K. Lee, S. E. Lee and J. K. Hwang, "Cornus kousa F. Buerger ex Miquel Increases Glucose Uptake through Activation of Peroxisome ProliferatorActivated Receptor $\gamma$ and Insulin Sensitization," Journal of Ethnopharmacology, Vol. 133, No. 2, 2011, pp. 803809. doi:10.1016/j.jep.2010.11.007

[84] M. A. Jayasri, A. Radha and T. L. Mathew, " $\alpha$-Amylase and $\alpha$-Glucosidase Inhibitory Activity of Costus pictus D. Don in the Management of Diabetes," Journal of Herbal Medicine and Toxicology, Vol. 3, No. 1, 2009, pp. 91-94.

[85] H.-S. Lee, "Cuminaldehyde: Aldose Reductase and $\alpha$ Glucosidase Inhibitor Derived from Cuminum cyminum L. Seeds," Journal of Agricultural and Food Chemistry, Vol. 53, No. 7, 2005, pp. 2446-2450. doi:10.1021/jf048451g

[86] D. Karthik and S. Ravikumar, "Characterization of the Brain Proteome of Rats with Diabetes Mellitus through Two-Dimensional Electrophoresis and Mass Spectrometry," Brain Research, Vol. 1371, 2011, pp. 171-179. doi:10.1016/j.brainres.2010.11.066

[87] D. Karthik and S. Ravikumar, "Proteome and Phytochemical Analysis of Cynodon dactylon Leaves Extract and Its Biological Activity in Diabetic Rats," Biomedicine \& Preventive Nutrition, Vol. 1, No. 1, 2011, pp. 49-56. doi:10.1016/j.bionut.2010.09.001

[88] D. Kuate, B. C. Etoundi, J. L. Ngondi and J. E. Oben, "Effects of Dichrostachys glomerata spice on Cardiovascular Diseases Risk Factors in Normoglycemic and Type 2 Diabetic Obese Volunteers," Food Research International, Vol. 44, No. 5, 2011, pp. 1197-1202. doi:10.1016/j.foodres.2010.09.037

[89] S. Kumar, V. Kumar and O. Prakash, "Antidiabetic, Hypolipidemic and Histopathological Analysis of Dillenia indica (L.) Leaves Extract on Alloxan Induced Diabetic Rats," Asian Pacific Journal of Tropical Medicine, Vol. 4, No. 5, 2011, pp. 347-352. doi:10.1016/S1995-7645(11)60101-6

[90] K. L. Ooi, T. S. T. Muhammad, M. L. Tan and S. F. Sulaiman, "Cytotoxic, Apoptotic and Anti- $\alpha$-Glucosidase Activities of 3,4-di-O-Caffeoyl Quinic Acid, an Antioxidant Isolated from the Polyphenolic-Rich Extract of Elephantopus mollis Kunth.," Journal of Ethnopharmacology, Vol. 135, No. 3, 2011, pp. 685-695. doi:10.1016/j.jep.2011.04.001

[91] T. Ieyama, M. D. P. T. Gunawan-Puteri and J. Kawabata, " $\alpha$-Glucosidase Inhibitors from the Bulb of Eleutherine americana," Food Chemistry, Vol. 128, No. 2, 2011, pp. 308-311. doi:10.1016/j.foodchem.2011.03.021

[92] S. Mahendran, S. Badami and V. Maithili, "Evaluation of Antidiabetic Effect of Embelin from Embelia ribes in Alloxan Induced Diabetes in Rats," Biomedicine \& Preventive Nutrition, Vol. 1, No. 1, 2011, pp. 25-31. 
doi:10.1016/j.bionut.2010.08.002

[93] S. V. Nampoothiri, A. Prathapan, O. L. Cherian, K. G. Raghu, V. V. Venugopalan and A. Sundaresan, "In Vitro Antioxidant and Inhibitory Potential of Terminalia bellerica and Embelica officinalis Fruits against LDL Oxidation and Key Enzymes Linked to Type 2 Diabetes," Food and Chemical Toxicology, Vol. 49, No. 1, 2011, pp. 125-131. doi:10.1016/j.fct.2010.10.006

[94] A. Toshima, T. Matsui, M. Noguchi, J. Qiu, K. Tamaya, Y. Miyata and T. Tanaka, "Identification of Alpha-Glucosidase Inhibitors from a New Fermented Tea Obtained by Tea-Rolling Processing of Loquat (Eriobotrya japonica) and Green Tea Leaves," Journal of the Science of Food and Agriculture, Vol. 90, No. 9, 2010, pp. 15451550. doi: $10.1002 /$ jsfa. 3983

[95] M. S. Deutschländer, N. Lall, M. Van de Venter and A. A. Hussein, "Hypoglycemic Evaluation of a New Triterpene and Other Compounds Isolated from Euclea undulata Thunb. var. myrtina (Ebenaceae) Root Bark," Journal of Ethnopharmacology, Vol. 133, No. 3, 2011, pp. 10911095. doi:10.1016/j.jep.2010.11.038

[96] B. Venkanna Babu, K. M. Prabhu and P. S. Murthy, "Studies on the Hypoglycemic Activity of the Bark of Ficus bengalensis Employing Alloxan Recovered Rabbits," Diabetes Bulletin, Vol. 7, 1987, pp. 105-108.

[97] N. Suksomboon, N. Poolsup, S. Boonkaew and C. C. Suthisisang, "Meta-Analysis of the Effect of Herbal Supplement on Glycemic Control in Type 2 Diabetes," Journal of Ethnopharmacology, Vol. 137, No. 3, 2011, pp. 1328-1333. doi:10.1016/j.jep.2011.07.059

[98] A. Kumari, V. K. Singh, J. Fitter, T. Polen and A. M. Kayastha, " $\alpha$-Amylase from Germinating Soybean ( Glycine $\max$ ) Seeds-Purification, Characterization and Sequential Similarity of Conserved and Catalytic Amino Acid Residues," Phytochemistry, Vol. 71, No. 14-15, 2010, pp. 1657-1666.

[99] K. Y. Kim, K. A. Nam, H. Kurihara and S. M. Kim, "Potent $\alpha$-Glucosidase Inhibitors Purified from the Red Alga Grateloupia elliptica," Phytochemistry, Vol. 69, No. 16, 2008, pp. 2820-2825. doi:10.1016/j.phytochem.2008.09.007

[100] A. B. Ali Ahmed, A. S. Rao and M. V. Rao, "In Vitro Callus and in Vivo Leaf Extract of Gymnema sylvestre stimulate $\beta$-Cells Regeneration and Anti-Diabetic Activity in Wistar Rats," Phytomedicine, Vol. 17, No. 13, 2010, pp. 1033-1039. doi:10.1016/j.phymed.2010.03.019

[101] K. M. Ramkumar, P. Vanitha, C. Uma, N. Suganya, E. Bhakkiyalakshmi and J. Sujatha, "Antidiabetic Activity of Alcoholic Stem Extract of Gymnema montanum in Streptozotocin-Induced Diabetic Rats," Food and Chemical Toxicology, Vol. 49, No. 12, 2011, pp. 3390-3394. doi:10.1016/j.fct.2011.09.027

[102] R. Galletto, V. L. Dias Siqueira, E. B. Ferreira, A. J. B. Oliveira and R. B. Bazotte, "Absence of Antidiabetic and Hypolipidemic Effect of Gymnema sylvestre in NonDiabetic and Alloxan-Diabetic Rats," Brazilian Archives of Biology and Technology, Vol. 47, No. 4, 2004, pp. 545-551. doi:10.1590/S1516-89132004000400007

[103] T. T. Wu, X. T. Zhou, Y. F. Deng, Q. Jing, M. Li, L. J.
Yuan, "In vitro studies of Gynura divaricata (L.) DC Extracts as Inhibitors of Key Enzymes Relevant for Type 2 Diabetes and Hypertension," Journal of Ethnopharmacology, Vol. 136, No. 2, 2011, pp. 305-308. doi:10.1016/j.jep.2011.04.059

[104] G. Kumar, G. S. Banu, A. G. Murugesan and M. R. Pandian, "Antihyperglycemic and Antiperoxidative Effect of Helicteres igora L. Bark Extracts in Streptozotocin-Induced Diabetic Rats," Journal of Applied Biomedicine, Vol. 5, 2007, pp. 97-104.

[105] C. Seifarth, L. Littmann, Y. Resheq, S. Rössner, A. Goldwich, N. Pangratz, F. Kerek, A. Steinkasserer and E. Zinser, "MCS-18, a Novel Natural Plant Product Prevents Autoimmune Diabetes," Immunology Letters, Vol. 139, No. 1-2, 2011, pp. 58-67.

[106] Özgür Devrim Can, Yusuf Öztürk, Nilgün Öztürk, Gianni Sagratini, Massimo Ricciutelli, Sauro Vittori, Filippo Maggi, "Effects of treatment with St. John's Wort on Blood Glucose Levels and Pain Perceptions of Streptozotocin-Diabetic Rats," Fitoterapia, Vol. 82, No. 4, 2011, 576-584. doi:10.1016/j.fitote.2011.01.008

[107] N. Orhan, A. Berkkan, D. D. Orhan, M. Aslan and F. Ergun, "Effects of Juniperus oxycedrus ssp. Oxycedrus on Tissue Lipid Peroxidation, Trace Elements ( $\mathrm{Cu}, \mathrm{Zn}, \mathrm{Fe})$ and Blood Glucose Levels in Experimental Diabetes," Journal of Ethnopharmacology, Vol. 133, No. 2, 2011, pp. 759-764. doi:10.1016/j.jep.2010.11.002

[108] Q.-H. Yang, Y. Liang, Q. Xu, Y. Zhang, L. Xiao and L.-Y. Si, "Protective Effect of Tetramethylpyrazine Isolated from Ligusticum chuanxiong on Nephropathy in Rats with Streptozotocin-Induced Diabetes," Phytomedicine, Vol. 18, No. 13, 2011, pp. 1148-1152.

doi:10.1016/j.phymed.2011.05.003

[109] H.-Q. Dong, M. Li, F. Zhu, F.-L. Liu and J.-B. Huang, "Inhibitory Potential of Trilobatin from Lithocarpus polystachyus Rehd against $\alpha$-Glucosidase and $\alpha$-Amylase Linked to Type 2 Diabetes," Food Chemistry, Vol. 130, No. 2, 2012, pp. 261-266. doi:10.1016/j.foodchem.2011.07.030

[110] S.-Z. Hou, S.-X. Chen, S. Huang, D.-X. Jiang, C.-J. Zhou, C.-Q. Chen, Y.-M. Liang and X.-P. Lai, "The Hypoglycemic Activity of Lithocarpus polystachyus Rehd. Leaves in the Experimental Hyperglycemic Rats," Journal of Ethnopharmacology, Vol. 138, No. 1, 2011, pp. 142-149. doi:10.1016/j.jep.2011.08.067

[111]A. Andrade-Cetto, "Inhibition of Gluconeogenesis by Malmea depressa Root," Journal of Ethnopharmacology, Vol. 137, No. 1, 2011, pp. 930-933. doi:10.1016/j.jep.2011.06.028

[112] A. Andrade-Cetto and E. Martínez-Zurita, A. Soto-Constantino, M. C. Revilla-Monsalve and H. Wiedenfeld, "Chronic Hypoglycemic Effect of Malmea depressa Root on n5-Streptozotocin Diabetic Rats," Journal of Ethnopharmacology, Vol. 116, No. 2, 2008, pp. 358-362. doi:10.1016/j.jep.2007.11.043

[113] A. A. Elberry, F. M. Harraz, S. A. Ghareib, S. A. Gabr, A. A. Nagy and E. Abdel-Sattar, "Methanolic Extract of Marrubium vulgare Ameliorates Hyperglycemia and Dyslipidemia in Streptozotocin-Induced Diabetic Rats," 
International Journal of Diabetes Mellitus, 2013 (in press).

[114] A. Fuangchan, P. Sonthisombat, T. Seubnukarn, R. Chanouan, P. Chotchaisuwat, V. Sirigulsatien, K. Ingkaninan, P. Plianbangchang and S. T. Haines, "Hypoglycemic Effect of Bitter Melon Compared with Metformin in Newly Diagnosed Type 2 Diabetes Patients," Journal of Ethnopharmacology, Vol. 134, No. 2, 2011, pp. 422-428. doi:10.1016/j.jep.2010.12.045

[115] A. Blum, C. Loerz, H.-J. Martin, C. A. Staab-Weijnitz and E. Maser, "Momordica charantia Extract, a Herbal Remedy for Type 2 Diabetes, Contains a Specific $11 \beta$-Hydroxysteroid Dehydrogenase Type 1 Inhibitor," The Journal of Steroid Biochemistry and Molecular Biology, Vol. 128, No. 1-2, 2012, pp. 51-55.

[116] D. S. Kumar, K. V. Sharathnath, P. Yogeswaran, A. Harani, K. Sudhakar and P. S. D. Banji, "A Medicinal Potency of Momordica charantia," International Journal of Pharmaceutical Sciences Review and Research, Vol. 1, No. 2, 2010, pp. 95-100.

[117] A. Tongia, S. K. Tomgia and M. Dave, "Phytochemical Determination and Extraction of Momordica charantia fruit and Its Hypoglycemic Potentiation of Oral Hypoglycemic Drugs in Diabetes Mellitus (NIDDM)," Indian Journal of Physiology and Pharmacology, Vol. 48, No. 2, 2004, pp. 241-244.

[118] B. Nickavar and G. Mosazadeh, "Influence of Three Morus Species Extracts on $\alpha$-Amylase Activity," Iranian Journal of Pharmaceutical Research, Vol. 8, No. 2, 2009, pp. 115-119.

[119] Y.-G. Li, D.-F. Ji, S. Zhong, Z.-Q. Lv, T.-B. Lin, S. Chen and G.-Y. Hu, "Hybrid of 1-Deoxynojirimycin and Polysaccharide from Mulberry Leaves Treat Diabetes Mellitus by Activating PDX-1/Insulin-1 Signaling Pathway and Regulating the Expression of Glucokinase, Phosphoenolpyruvate Carboxykinase and Glucose-6-Phosphatase in Alloxan-Induced Diabetic Mice," Journal of Ethnopharmacology, Vol. 134, No. 3, 2011, pp. 961-970. doi:10.1016/j.jep.2011.02.009

[120] G. T. Volpato, I. M. P. Calderon, S. Sinzato, K. E. Campos, M. V. C. Rudge and D. C. Damasceno, "Effect of Morus nigra Aqueous Extract Treatment on the Maternal-Fetal Outcome, Oxidative Stress Status and Lipid Profile of Streptozotocin-Induced Diabetic Rats," Journal of Ethnopharmacology, Vol. 138, No. 3, 2011, pp. 691696. doi:10.1016/j.jep.2011.09.044

[121] S. O. Majekodunmi, A. A. Oyagbemi, S. Umukoro and O. A. Odeku, "Evaluation of the Anti-Diabetic Properties of Mucuna pruriensseed Extract," Asian Pacific Journal of Tropical Medicine, Vol. 4, No. 8, 2011, pp. 632-636. doi:10.1016/S1995-7645(11)60161-2

[122] H. Yankuzo, Q. U. Ahmed, R. I. Santosa, S. F. U. Akter and N. A. Talib, "Beneficial Effect of the Leaves of Murraya koenigii (Linn.) Spreng (Rutaceae) on Diabetes-Induced Renal Damage in Vivo," Journal of Ethnopharmacology, Vol. 135, No. 1, 2011, pp. 88-94. doi:10.1016/j.jep.2011.02.020

[123] U. D. Palanisamy, L. T. Ling, T. Manaharan and D. Appleton, "Rapid Isolation of Geraniin from Nephelium lap- paceum Rind Waste and Its Anti-Hyperglycemic Activity," Food Chemistry, Vol. 127, No. 1, 2011, pp. 21-27. doi:10.1016/j.foodchem.2010.12.070

[124] U. Palanisamy, T. Manaharan, L. L. Teng, A. K. C. Radhakrishnan, T. Subramaniam and T. Masilamani, "Rambutan Rind in the Management of Hyperglycemia," Food Research International, Vol. 44, No. 7, 2011, pp. 22782282.

[125] E. K. Dilip Kumar and G. R. Janardhana, “Antidiabetic Activity of Alcoholic Stem Extract of Nervilia plicata in Streptozotocin-Nicotinamide Induced Type 2 Diabetic Rats," Journal of Ethnopharmacology, Vol. 133, No. 2, 2011, pp. 480-483. doi:10.1016/j.jep.2010.10.025

[126] Y.-N. Huang, Y.-L. Zhao, X.-L. Gao, Z.-F. Zhao, Z. Jing, W.-C. Zeng, R. Yang, R. Peng, T. Tong, L.-F. Wang, J.-Q. Cen and H. Gao, "Intestinal $\alpha$-Glucosidase Inhibitory Activity and Toxicological Evaluation of Nymphaea stellata Flower Extract," Journal of Ethnopharmacology, Vol. 131, No. 2, 2010, pp. 306-312. doi:10.1016/j.jep.2010.06.035

[127] R. Patil, R. Patil, B. Ahirwar and D. Ahirwar, "Isolation and Characterization of Anti-Diabetic Component (Bioactivity-Guided Fractionation) from Ocimum sanctum L. (Lamiaceae) Aerial Part," Asian Pacific Journal of Tropical Medicine, Vol. 4, No. 4, 2011, pp. 278-282. doi:10.1016/S1995-7645(11)60086-2

[128] J. Xu, Y. Wang, D.-S. Xu, K.-F. Ruan, Y. Feng and S. Wang, "Hypoglycemic Effects of MDG-1, a Polysaccharide Derived from Ophiopogon japonicas, in the $o b / o b$ Mouse Model of Type 2 Diabetes Mellitus," International Journal of Biological Macromolecules, Vol. 49, No. 4, 2011, pp. 657-662. doi:10.1016/j.ijbiomac.2011.06.026

[129] S.-W. Hahm, J. Park and Y.-S. Son, "Opuntia humifusa Stems Lower Blood Glucose and Cholesterol Levels in Streptozotocin-Induced Diabetic Rats," Nutrition Research, Vol. 31, No. 6, 2011, pp. 479-487. doi:10.1016/j.nutres.2011.05.002

[130] L. Y. Zhao, Q. J. Lan, Z. C. Huang, L. J. Ouyang and F. H. Zeng, "Antidiabetic Effect of a Newly Identified Component of Opuntia dillenii Polysaccharides," Phytomedicine, Vol. 18, No. 8-9, 2011, pp. 661-668. doi:10.1016/i.phymed.2011.01.001

[131] A. Andrade-Cetto and H. Wiedenfeld, "Anti-Hyperglycemic Effect of Opuntia streptacantha Lem," Journal of Ethnopharmacology, Vol. 133, No. 2, 2011, pp. 940-943. doi:10.1016/j.jep.2010.11.022

[132] A. M. Guzman-Partida, O. Jatomea-Fino, M. R. RoblesBurgueño, M. Ortega-Nieblas, L. Vazquez-Moreno, "Characterization of $\alpha$-Amylase Inhibitor from Palo $\mathrm{fi}$ erro Seeds," Plant Physiology and Biochemistry, Vol. 45, No. 9, 2007, pp. 711-715. doi:10.1016/j.plaphy.2007.07.002

[133] N. Angelova, H.-W. Kong, R. Van Der Heijden, S.-Y. Yang, Y. H. Choi, H. K. Kim, M. Wang, T. Hankemeier, J. Van Der Greef, G. W. Xu and R. Verpoorte, "Recent Methodology in the Phytochemical Analysis of Ginseng," Phytochemical Analysis, Vol. 19, No. 1, 2008, pp. 2-16. doi:10.1002/pca.1049

[134] Y.-Z. Xiang, H.-C. Shang, X.-M. Gao and B.-L. Zhang, 
"A Comparison of the Ancient Use of Ginseng in Traditional Chinese Medicine with Modern Pharmacological Experiments and Clinical Trials," Phytotherapy Research, Vol. 22, No. 7, 2008, pp. 851-858. doi:10.1002/ptr.2384

[135] Z. G. Wu, J. Z. Luo and L. G. Luo, "American Ginseng Modulates Pancreatic Beta Cell Activities," Chinese Medicine, Vol. 2, No. 11, 2007, pp. 1-5.

[136] V. Vuksan, J. L. Sievenpiper, V. Y. Y. Koo and T. Francis, "American Ginseng (Panax quinquefolius L.) Reduces Postprandial Glycemia in Nondiabetic Subjects and Subjects with Type 2 Diabetes Mellitus," Archives of Internal Medicine, Vol. 160, No. 7, 2000, pp. 1009-1013. doi:10.1001/archinte.160.7.1009

[137] T. Manaharan, L. L. Teng, D. Appleton, C. H. Ming, T. Masilamani and U. D. Palanisamy, "Antioxidant and Antiglycemic Potential of Peltophorum pterocarpum Plant Parts," Food Chemistry, Vol. 129, No. 4, 2011, pp. 13551361. doi:10.1016/j.foodchem.2011.05.041

[138] H. Ali, P. J. Houghton and A. Soumyanath, “ $\alpha$-Amylase Inhibitory Activity of Some Malaysian Plants Used to Treat Diabetes; With Particular Reference to Phyllanthus amarus," Journal of Ethnopharmacology, Vol. 107, No. 3, 2006, pp. 449-455. doi:10.1016/j.jep.2006.04.004

[139] P. Bansal, P. Paul, J. Mudgal, P. G. Nayak, S. T. Pannakal, K. I. Priyadarsini and M. K. Unnikrishnan, "Antidiabetic, Antihyperlipidemic and Antioxidant Effects of the Flavonoid Rich Fraction of Pilea microphylla (L.) in High Fat Diet/Streptozotocin-Induced Diabetes in Mice," Experimental and Toxicologic Pathology, Vol. 64, No. 6, 2013, pp. 651-658.

[140] R. R. Saddala, L. Thopireddy, N. Ganapathi and S. R. Kesireddy, "Regulation of Cardiac Oxidative Stress and Lipid Peroxidation in Streptozotocin-Induced Diabetic Rats Treated with Aqueous Extract of Pimpinella tirupatiensis Tuberous Root," Experimental and Toxicologic Pathology,2011 (online).

[141] Y.-M. Kim, Y.-K. Jeong, M.-H. Wang, W.-Y. Lee and H.-I. Rhee, "Inhibitory Effect of Pine Extract on $\alpha$-Glucosidase Activity and Postprandial Hyperglycemia," Nutrition, Vol. 21, No. 8, 2005, pp. 756-761. doi:10.1016/j.nut.2004.10.014

[142] V. Vadivel and H. K. Biesalski, "Contribution of Phenolic Compounds to the Antioxidant Potential and Type II Diabetes Related Enzyme Inhibition Properties of Pongamia pinnata L. Pierre Seeds," Process Biochemistry, Vol. 46, No. 10 , 2011, pp. 1973-1980. doi:10.1016/j.procbio.2011.07.007

[143] M.-I. Kotb El-Sayed, "Effects of Portulaca oleracea L. seeds in Treatment of Type-2 Diabetes Mellitus Patients as Adjunctive and Alternative Therapy," Journal of Ethnopharmacology, Vol. 137, No. 1, 2011, pp. 643-651. doi:10.1016/j.jep.2011.06.020

[144] C. George, A. Lochner and B. Huisamen, "The Efficacy of Prosopis glandulosa as Antidiabetic Treatment in Rat Models of Diabetes and Insulin Resistance," Journal of Ethnopharmacology, Vol. 137, No. 1, 2011, pp. 298-304. doi:10.1016/j.jep.2011.05.023

[145] S. Soman, C. Rajamanickam, A. A. Rauf and M. Indira, "Beneficial Effects of Psidium guajava Leaf Extract on
Diabetic Myocardium," Experimental and Toxicologic Pathology, Vol. 65, No. 1-2, 2013, pp. 91-95.

[146] V. K. Kondeti, K. R. Badri, D. R. Maddirala,S. K. M. Thur, S. S. Fatima, R. B. Kasetti and C. A. Rao, "Effect of Pterocarpus santalinus Bark, on Blood Glucose, Serum Lipids, Plasma Insulin and Hepatic Carbohydrate Metabolic Enzymes in Streptozotocin-Induced Diabetic Rats," Food and Chemical Toxicology, Vol. 48, No. 5, 2010, pp. 1281-1287. doi:10.1016/j.fct.2010.02.023

[147] S. P. Dhanabal, C. K. Kokate, M. Ramanathan, E. P. Kumar and B. Suresh, "Hypoglycemic Activity of Petrocarpus marsupium Roxb.," Phytotherapy Research, Vol. 20, No. 1, 2006, pp. 4-8. doi:10.1002/ptr.1819

[148] M. C. Joshi, M. Dorababu, T. Prabha, M. M. Kumar and R. K. Goel, "Effects of Pterocarpus marsupium on NIDDM-Induced Rat Gastric Ulceration and Mucosal Offensive and Defensive Factors," Indian Journal of Pharmacology, Vol. 36, No. 5, 2004, pp. 296-302.

[149] K. H. Wong, G. Q. Li, K. M. Li, V. Razmovski-Naumovski and K. Chan, "Kudzu Root: Traditional Uses and Potential Medicinal Benefits in Diabetes and Cardiovascular Diseases," Journal of Ethnopharmacology, Vol. 134, No. 3, 2011, pp. 584-607. doi:10.1016/j.jep.2011.02.001

[150] G. G. Adams, S. Imran, S. Wang, A. Mohammad, S. Kok, D. A. Gray, G. A. Channell, G. A. Morris and S. E. Harding, "The Hypoglycemic Effect of Pumpkins as AntiDiabetic and Functional Medicines," Food Research International, Vol. 44, No. 4, 2011, pp. 862-867. doi:10.1016/j.foodres.2011.03.016

[151] M. Makni, H. Fetoui, N. K. Gargouri, El M. Garoui and N. Zeghal, "Antidiabetic Effect of Flax and Pumpkin Seed Mixture Powder: Effect on Hyperlipidemia and Antioxidant Status in Alloxan Diabetic Rats," Journal of Diabetes and its Complications, Vol. 25, No. 5, 2011, pp. 339-345. doi:10.1016/j.jdiacomp.2010.09.001

[152] K. S. Babu, A. K. Tiwari, P. V. Srinivas, A. Z. Ali, B. C. Raju and J. M. Rao, "Yeast and Mammalian $\alpha$-Glucosidase Inhibitory Constituents from Himalayan Rhubarb Rheum emodi Wall.ex Meisson," Bioorganic \& Medicinal Chemistry Letters, Vol. 14, No. 14, 2004, pp. 3841-3845. doi:10.1016/j.bmcl.2004.04.062

[153] R. Sedaghat, M. Roghani, M. Ahmadi and F. Ahmadi, "Antihyperglycemic and Antihyperlipidemic Effect of Rumex patientia Seed Preparation in StreptozotocinDiabetic Rats," Pathophysiology, Vol. 18, No. 2, 2011, pp. 111-115. doi:10.1016/j.pathophys.2010.03.001

[154] S. Nakamura, K. Takahira, G. Tanabe, T. Morikawa, M. Sakano, K. Ninomiya, M. Yoshikawa, O. Muraoka and I. Nakanishi, "Docking and SAR Studies of Salacinol Derivatives as $\alpha$-Glucosidase Inhibitors," Bioorganic \& Medicinal Chemistry Letters, Vol. 20, No. 15, 2010, pp. 4420-4423. doi:10.1016/j.bmcl.2010.06.059

[155] X.-K. Zheng, L. Zhang, W.-W. Wang, Y.-Y. Wu, Q.-B. Zhang and W.-S. Feng, "Anti-Diabetic Activity and Potential Mechanism of Total Flavonoids of Selaginella tamariscina (Beauv.) Spring in Rats Induced by High Fat Diet and Low Dose STZ," Journal of Ethnopharmacology, Vol. 137, No. 1, 2011, pp. 662-668. doi:10.1016/j.jep.2011.06.018 
[156] N. C. Habib, S. M. Honoré, S. B. Genta and S. S. Sánchez, "Hypolipidemic Effect of Smallanthus sonchifolius (yacon) Roots on Diabetic Rats: Biochemical Approach," Chemico-Biological Interactions, Vol. 194, No. 1, 2011, pp. 31-39. doi:10.1016/j.cbi.2011.08.009

[157] G. R. Gandhi, S. Ignacimuthu, M. G. Paulraj and P. Sasikumar, "Antihyperglycemic Activity and Antidiabetic Effect of Methyl Caffeate Isolated from Solanum torvum Swartz. Fruit in Streptozotocin Induced Diabetic Rats," European Journal of Pharmacology, Vol. 670, No. 2-3, 2011, pp. 623-631. doi:10.1016/j.ejphar.2011.09.159

[158] J.-S. Kim, T. K. Hyun and M.-J. Kim, "The Inhibitory Effects of Ethanol Extracts from Sorghum, Foxtail Millet and Proso Millet on $\alpha$-Glucosidase and $\alpha$-Amylase Activities," Food Chemistry, Vol. 124, No. 4, 2011, pp. 1647-1651. doi:10.1016/j.foodchem.2010.08.020

[159] R. Mogra and V. Dashora, "Exploring the Use of Stevia rebaudiana as a Sweetener in Comparison with Other Sweeteners," Journal of Human Ecology, Vol. 25, No. 2, 2009, pp. 117-120.

[160] M. H. Sumon, M. Mostofa, M. S. Jahan, M. E. H. Kayesh and M. A. Haque, "Comparative Efficacy of Powdered form of Stevia (Stevia rebaudiana Bertoni) Leaves and Glimepiride in Induced Diabetec Rats," Bangladesh Journal of Veterinary Medicine, Vol. 6, No. 2, 2008, pp. 211-215.

[161] S. Gregersen, P. B. Jeppesen, J. J. Holst and K. Hermansen, "Antihyperglycemic Effects of Stevioside in Type 2 Diabetic Subjects," Metabolism, Vol. 53, No. 1, 2004, pp. 73-76. doi:10.1016/j.metabol.2003.07.013

[162] S. Banerjee, T. K. Sur, S. Mandal, P. C. Das and S. Sikdar, "Assessment of the Anti-Inflammatory Effects of Swertia chirata in Acute and Chronic Experimental Models in Male Albino Rats," Indian Journal of Pharmaceutical, Vol. 32, 2000, pp. 21-24.

[163] C. Sunil, S. Ignacimuthu and P. Agastian, "Antidiabetic Effect of Symplocos cochinchinensis (Lour.) S. Moore. in Type 2 Diabetic Rats," Journal of Ethnopharmacology, Vol. 134, No. 2, 2011, pp. 298-304. doi:10.1016/j.jep.2010.12.018

[164] K. S. De Bona, L. P. Bellé, P. E. R. Bittencourt, G. Bonfanti, L. O. Cargnelluti, V. C. Pimentel, A. R. Ruviaro, M. R. C. Schetinger, T. Emanuelli and M. B. Moretto, "Erythrocytic Enzymes and Antioxidant Status in People with Type 2 Diabetes: Beneficial Effect of Syzygium cumini Leaf Extract in Vitro," Diabetes Research and Clinical Practice, Vol. 94, No. 1, 2011, pp. 84-90. doi:10.1016/i.diabres.2011.06.008

[165] Z. P. Ruan, L. L. Zhang and Y. M. Lin, "Evaluation of the Antioxidant Activity of Syzygium cumini Leaves," Molecules, Vol. 13, No. 10, 2008, pp. 2545-2556. doi:10.3390/molecules13102545

[166] J. R. Shinde, T. Taldone, M. Barletta, N. Kunaparaju, B. $\mathrm{Hu}, \mathrm{S}$. Kumar and J. Placido, " $\alpha$-Glucosidase Inhibitory Activity of Syzygium cumini (Linn.) Skeels Seed Kernel in Vitro and Goto-Kakizaki (GK) Rats," Carbohydrate Research, Vol. 343, No. 7, 2008, pp. 1278-1281. doi:10.1016/j.carres.2008.03.003

[167] A. Kumar, R. IIavarasan, T. Jayachandran, M. Deecara- man, P. Aravindan, N. Padmanabhan and M. R. V. Krishan, "Anti-Diabetic Activity of Syzygium cumini and Its Isolated Compound against Streptozotocin-Induced Diabetic Rats," Journal of Medicinal Plants Research, Vol. 2, No. 9, 2008, pp. 246-249.

[168] S. Ramachandran, A. Rajasekaran and K. T. M. Kumar, "Antidiabetic, Antihyperlipidemic and Antioxidant Potential of Methanol Extract of Tectona grandis Flowers in Streptozotocin Induced Diabetic Rats," Asian Pacific Journal of Tropical Medicine, Vol. 4, No. 8, 2011, pp. 624-631. doi:10.1016/S1995-7645(11)60160-0

[169] R. C. R. Latha and P. Daisy, "Insulin-Secretagogue, Antihyperlipidemic and Other Protective Effects of Gallic Acid Isolated from Terminalia bellerica Roxb. in Streptozotocin-Induced Diabetic Rats," Chemico-Biological Interactions, Vol. 189, No. 1-2, 2011, pp. 112-118.

[170] A. Umar, Q. U. Ahmed, B. Y. Muhammad, B. B. S. Dogarai and S. Z. Bt. Mat Soad, "Anti-Hyperglycemic Activity of the Leaves of Tetracera scandens Linn. Merr. (Dilleniaceae) in Alloxan Induced Diabetic Rats," Journal of Ethnopharmacology, Vol. 131, No. 1, 2010, pp. 140-145. doi:10.1016/j.jep.2010.06.016

[171] A. Domingues, A. Sartri, M. A. Golim, L. M. M. Valente, L. C. da Rosa, L. L. W. Ishikawa, A. C. Siani and R. M. Viero, "Prevention of Experimental Diabetes by Uncaria tomentosa Extract: Th2 Polarization, Regulatory T Cell Preservation or Both?" Journal of Ethnopharmacology, Vol. 137, No. 1, 2011, pp. 635-642.

doi:10.1016/j.jep.2011.06.021

[172] L. Wang, X. T. Zhang, H. Y. Zhang, H. Y. Yao and H. Zhang, "Effect of Vaccinium bracteatum Thunb. Leaves Extract on Blood Glucose and Plasma Lipid Levels in Streptozotocin-Induced Diabetic Mice," Journal of Ethnopharmacology, Vol. 130, No. 3, 2010, pp. 465-469. doi:10.1016/j.jep.2010.05.031

[173] K. W. Ong, A. Hsu, L. X. Song, D. J. Huang and B. K. H. Tan, "Polyphenols-Rich Vernonia amygdalina Shows Anti-Diabetic Effects in Streptozotocin-Induced Diabetic Rats," Journal of Ethnopharmacology, Vol. 133, No. 2, 2011, pp. 598-607. doi:10.1016/j.jep.2010.10.046

[174] R. Gonçalves, N. Mateus and V. de Freitas, "Inhibition of $\alpha$-Amylase Activity by Condensed Tannins," Food Chemistry, Vol. 125, No. 2, 2011, pp. 665-672. doi:10.1016/j.foodchem.2010.09.061

[175] L. Zhang, S. Hogan, J. R. Li, S. Sun, C. Canning, S. Jian Zheng and K. Q. Zhou, "Grape Skin Extract Inhibits Mammalian Intestinal $\alpha$-Glucosidase Activity and Suppresses Postprandial Glycemic Response in Streptozocin-Treated Mice," Food Chemistry, Vol. 126, No. 2, 2011, pp. 466-471. doi:10.1016/j.foodchem.2010.11.016

[176] K. R. Shanmugam, K. Mallikarjuna, K. Nishanth, C. H. Kuo and K. S. Reddy, "Protective Effect of Dietary Ginger on Antioxidant Enzymes and Oxidative Damage in Experimental Diabetic Rat Tissues," Food Chemistry, Vol. 124, No. 4, 2011, pp. 1436-1442. doi:10.1016/i.foodchem.2010.07.104

[177] C. G. Michel, D. I. Nesseem and M. F. Ismail, "AntiDiabetic Activity and Stability Study of the Formulated Leaf Extract of Zizyphus spina-christi (L.) Willd with the 
Influence of Seasonal Variation," Journal of Ethnopharmacology, Vol. 133, No. 1, 2011, pp. 53-62.

doi:10.1016/j.jep.2010.09.001

[178] J. El Ghoul, N. Ghanem-Boughanmi and M. Ben-Attia, "Biochemical Study on the Protective Effect of Ethanolic Extract of Zygophyllum album on Streptozotocin-Induced Oxidative Stress and Toxicity in Mice," Biomedicine \& Preventive Nutrition, Vol. 1, No. 2, 2011, pp. 79-83. doi:10.1016/j.bionut.2010.10.001

[179] H. Matsuda, N. Nishida and M. Yoshikawa, "Antidiabetic Principles of Natural Medicines. V. Aldose Reductase Inhibitors from Myrcia multiflora DC. (2): Structures of Myrciacitrins III, IV, and V," Chemical and Pharmaceutical Bulletin, Vol. 50, No. 3, 2002, pp. 429-431. doi:10.1248/cpb.50.429

[180] T. Tsutsumi, S. Kobayashi, Y. Y. Liu and H. Kontani, "Anti-Hyperglycemic Effect of Fangchinoline Isolated from Stephani tetrandra Radix in Streptozotocin-Diabetic
Mice," Biological and Pharmaceutical Bulletin, Vol. 26, No. 3, 2003, pp. 313-317. doi:10.1248/bpb.26.313

[181] T. Kiyoteru, T. Shinichi, K. Junichi, Y. Shunivhi, L. Kazuo, W. Kinzo, J. Samisoni, V. Taraiasi and A. Bill, "Hyperglycemia Inhibitor and Method for Producing the Same," Japanese Patent JP2005132837, 2005.

[182] L. Costantino, L. Raimondi, R. Pirisino, T. Brunetti, P. Pessotto, F. Giannessi, A. P. Lins, D. Barlocco, L. Antolini and S. A El-Abady, "Isolation and Pharmacological Activities of the Tecoma stans alkaloids," Il Farmaco, Vol.58, No. 9, 2003, pp. 781-785. doi:10.1016/S0014-827X(03)00133-2

[183] A. Chopra, M. Saluja and G. Tillu, “Ayurveda-Modern Medicine Interface: A Critical Appraisal of Studies of Ayurvedic Medicines to Treat Osteoarthritis and Rheumatoid Arthritis," Journal of Ayurveda \& Integrative Medicine, Vol. 1, No. 3, 2010, pp. 190-198. doi:10.4103/0975-9476.72620 